PPPL -2686

DE9 0008193

\title{
Loss of Apha-Like MeV Fusion Products from TFTR
}

\author{
S. J. Zweben, R. L. Boivin, M. Diesso, S.E. Hayes, H.W. Hendel, \\ H. Park, and J.D. Strachan \\ Princeton Plasma Physics Laboratory, Princeton University \\ Princeton, NJ 08543 USA
}

\begin{abstract}
A detailed comparison between the observed and expected loss of alpha-like $\mathrm{MeV}$ fusion products in TFTR is presented. The D-D fusion products (mainly the $1 \mathrm{MeV}$ triton) were measured with an 2-D imaging scintillation detector. The expected first-orbit loss was calculated with a simple Lorentz orbit code. In almost all cases the measured loss was consistent with the expected first-orbit loss model. Exceptions are noted for small major radius plasmas and during strong MHD activity.
\end{abstract}

\section{DISCLAIMER}

\begin{abstract}
This report was prepared as an scount of work sponsored by an asency of the United Sutes Government. Neither the United States Govetnment nor any agency thereof, nor any of their employees, makes any wasranty, exprets of inplied, or assumes any lezal liability or responsibility for the aceuracy. compteteness, or usefulness of any information, apparatus, product, or process disclowed, or represents that its use would aot iafringe privalely owned rights. Reference berein to any specific commercial product, process, or servioe by trade name, trademark, manulacturer, of otherwise daes not necessurily contitute or impiy its endorsement, recommendation, or favoring by the United Sintes Coverament or any arency thereor. The views and opinions of authors expecsed herein do mot necessurily stute or rellect those of the United States Government of any agency thereor.
\end{abstract}




\section{INTRODUCTION}

Good alpha-particle confinement will be necessary to obtain D-T Ignition in a tokamak, and accurate predictions of alpha losses will be needed for designing a fusion reactor first wall. This paper describes some recent experiments on TFTR which test our understanding of the "single-particle" energetic ion confinement obtained with the relatively small populations of D-D fusion products. This understanding can form a baseline for future study of the "collective" alpha instability and heating effects to be obtained with the $=1000$ times larger MeV ion populations expected for ignited D-T plasmas.

\subsection{Alpha Confinement Models}

The simplest axisymmetric, neoclassical, alpha particle confinement model predicts that (almost) all alphas which are confined on their first orbit will stay confined until they are thermalized, since energy drag dominates pitch angle scattering during the thermalization process $[1,2]$. Thus, alpha loss should become significant when the typical banana width " $\Delta$ " becomes comparable to or larger than about half the minor radius, or roughly:

$$
\Delta=q(R / a)^{1 / 2} \rho_{\text {tor }}>a / 2
$$

where $q$ is the magnetic safety factor, $R$ is the plasma major radius, $a$ is the plasma minor radius, and $p_{\text {tor }}$ is the toroidal gyroradius. Therefore, the main determinant of alpha confinement at a given toroidal field and aspect ratio (R/a) is the plasma current, I, which determines q. Thus for $3.5 \mathrm{MeV}$ alpha particles in TFTR with $\mathrm{R}=245 \mathrm{~cm}, \mathrm{a}=80 \mathrm{~cm}, \mathrm{~B}_{\text {tor }}=50 \mathrm{kG}$, and $\rho_{\text {tor }}=5 \mathrm{~cm}$, condition (1) occurs at about $I=1.5 \mathrm{MA}$. Therefore, first-orbit loss of alpha-like ions should be dominant below about $0.5 \mathrm{MA}$ and small above about $2 \mathrm{MA}$, as has been calculated previously in slightly different forms [1-5].

In fact, at the very highest currents typical of current designs for ignition tokamaks ( $>10 \mathrm{MA}$ ), the neoclassical axisymmetric alpha loss is 
negligible, and the dominant loss should be due to the small non-axisymmetric toroidal field (TF) ripple, which can cause up to $10-20 \%$ of the alpha-heating power to be lost to the walls [6-8]. This TF ripple loss is due to relatively slow diffusion of trapped ion orbits, and so should occur primarily at the point where the previously confined orbits first intersect the wall, i.e., near the outer equatorial plane [9].

These calculations of axisymmetric and TF ripple losses are fairly straightforward, since they are based on well-known vacuum magnetic field configurations. However, more recent models which calculate the loss due to internally generated non-axisymmetric perturbations resulting from magnetohydrodynamic (MHD) instabilities or small-scale turbulence [10-11] cannot yet be applied with such confidence, since the magnitudes and internal structure of these perturbations are not (and may never be) well known. Therefore, it seems appropriate to evaluate the alpha confinement experimentally.

\subsection{Previous Experimental Work}

Experimental tests of single-particle alpha confinement can be made by exploiting the similarity between the $3.5 \mathrm{MeV}$ D-T alphas and two of the charged fusion products routinely created in D-D plasmas. namely the 1 $\mathrm{MeV}$ triton and $3 \mathrm{MeV}$ proton. These two fusion products are similar to the $3.5 \mathrm{MeV}$ alphas with respect to their gyroradii (to within $10 \%$ ) and thermalization time $(\approx 0.5-1.0 \mathrm{sec})$, so that their confinemert and thermalization physics should be the same for almost any type of fusion product loss mechanism.

Two complementary experimental techniques have been used previously to measure alpha-like D-D fusion reaction products. The first is the "burnup" technique for confined triton detection, which measures the total confined triton population vs. time through the $14 \mathrm{MeV}$ neutrons created by the thermalizing tritons in their reactions with the deuterium background plasma. The other technique, used in this paper, is direct measurement of charged fusion product losses to the wall.

Pioneering work on triton (and $3 \mathrm{He}$ ) burnup in PLT and PEX [12] showed that in many cases the results were consistent with classical confinement 
ar.d thermalization (to within a factor of three uncertainty), but in some other cases there was an anomalously low burnup at low TF, possibly associated with large MHD activity. Enhanced MeV-ion losses associated with MHD "fishbone" activity was also observed on PBX [13]. More recently, triton burnup measurements on FT [14] and on JET at plasma currents up to 5 MA [15] have shown that global triton confinement and slowing-down are almost always consistent with the axisymmetric neoclassical model predictions, with possible exceptions being associated with increased MHD activity. Similar TFTR experiments have usually shown a burnup which is anomalously low by a factor of two [16], particularly when the triton slowing-down time is long in "supershots". Identification of possible anomalous loss mechanisms via the burnup technique tends to be difficult, since the $14 \mathrm{MeV}$ neutron measurements are volume averaged and have relatively poor time resolution ( $\approx 50 \mathrm{msec}$ ).

The complementary technique described in this paper directly measures the loss of charged fusion products to detectors located inside the vacuum vessel but outside the plasma boundary. This method can provide very localized space and time resolved measurements of the triton or proton losses $(<1 \mathrm{~cm},<10 \mu \mathrm{sec})$, but global triton confinement cannot easily be evaluated, since an integration of these losses over the whole wall area would be necessary.

Direct measurements of D-D fusion product losses were first made in low current tokamaks such as PLT [17,18] and ASDEX [19]. These experiments measured escaping $3 \mathrm{MeV}$ protons with silicon surface barrier (SSB) detectors in order to determine the deuterium ion temperature, which required good energy resolution but little or no pitch angle resolution.

Other previous measurements have detected the fusion products from D. ${ }^{3} \mathrm{He}$ reactions, namely, the $15 \mathrm{MeV}$ protons and the $3.7 \mathrm{MeV}$ alpha particles. Particularly relevant to the present experiment was a measurement of the energy and pitch angle distributions of escaping 3.7 $\mathrm{MeV}$ alpha-particles in PLT [20], which was made with a time-integrating plastic track detector in geometrical configuration similar to the one used here. In TFTR, measurements of the escaping $15 \mathrm{MeV}$ protons from the burnup of the $0.8 \mathrm{MeV}{ }^{3} \mathrm{He}$ ions produced by D-D reactions were made using an array of SSB detectors, with results approximately consistent with neoclassical predictions [21]. In JET, measurements of the $15 \mathrm{MeV}$ protons from ICRF-induced $\mathrm{D}-{ }^{3} \mathrm{He}$ reactions were made using a 
single-channel SSB detector, with striking results showing large bursts of lost protons associated with sawteeth during ICRF minority heating [22].

Conspicuously absent from the previous experimental work are direct measurements of alpha-like particle losses in the plasma current range 0.5 - 2.0 MA over which the first-orbit loss should vary from dominant to negligible. Also absent are direct measurements of the enhanced losses which were sometimes cbserved indirectly through anomalously low triton burnup.

The TFTR work in these directions began with a survey of possible alpha physics experiments [23], and with a prototype 4-channel Zns scintillation detector [24]. A second 2-D imaging prototype for measuring escaping tritons was operated on TFTR in 1987 [25]. That detector, despite its relativley poor time resolution and large soft $x$-ray background, did demonstrate some of the basic features of triton loss which are reproduced and extended in the present experiment.

The present paper describes the 2-D imaging detection system in Sec. 2 and the experimental results in Sec. 3 . The conclusions, including some tentative theoretical interpretations and directions for further experiments, are in Sec. 4.

\section{DETECTION SYSTEM}

This section describes the design and operation of the lost triton detection system on TFTR. Many of the general design considerations are similar to those discussed previously tor the 2-D prototype detector [25].

\subsection{Detector Configuration}

Figure 1 shows the arrangement of the lost triton detectors inside the TFTR vacuum vessel for the 1988 run. There were eight detectors installed, four of which are inserted from underneath the vessel along a poloidal arc (the "poloidal array"), and four of which are mounted inside the vessel in a square array (the "toroidal array"). All were fixed in position at the bottom of the vessel (the direction of the ion grad-B drift) during the entire 1988 run. 
The experimental results described in Sec. 3 come almost entirely from detector \#6, which was located about $90^{\circ}$ below the outer midplane (as was the prototype in Ref. 25). This detector was monitored most carefully because it had the largest signal levels, due to its relatively high optical efficiency. However, the results from the other triton/proton poloidal detectors (\#9 and \#11) were at least qualitatively similar, as discussed in Sec. 3.12.

\subsection{General Detector Design}

As shown in Figs. 2 and 3 , the tritons and/or protons enter a pinhole/slit aperture pair, pass through a thin aluminum foil, and then hit a scintillator screen mounted inside the light-tight detector box. The 2-D images of light from the scintillator screen are coupled out of the vacuum system by a relay lens and into an $8 \mathrm{~m}$ long coherent fiber bundle which runs to signal monitors in the neutron-shielded TFTR basement.

The \#6 detector was positioned so that the top of its box was $101 \mathrm{~cm}$ radially from the centers of the "RF limiters", comprising a pair of poloidal rings located $120^{\circ}$ and $170^{\circ}$ toroidally from the location of the poloidal array in the direction parallel to the plasma current. At this radial position the top of the box was $2 \mathrm{~cm}$ into the shadow or these limiters, which had a minor radius of $99 \mathrm{~cm}$ from a center at $\mathbf{R}=261 \mathrm{~cm}$. This shadowing was necessary in order to limit the heat flux to the triton probe box, but this position still allowed all relevant triton orbits to reach the detector aperture unobstructed by the RF limiters.

The scintillator itself was oriented so as to have a pitch angle detection range of $X=40^{\circ}-90^{\circ}$ with respect to the "co" direction of the toroidal field. Note that in this paper the pitch angle of the particle orbit $X$ is the angle of the particle velocity $v$ with respect to the local toroidal magnetic field at the detector, i. $\theta ., \cos X=v_{\text {tor }} / v$, (this angle is conveniently defined with respect to the fixed detector independently of the particular plasma current). This range was centered near the expected $=60^{\circ}$ pitch angles of the first-orbit losses by rotating the detector shaft about its axis $22.5^{\circ}$ from aperture-outward position into the "co" direction, so that the center of the scintillator corresponded to a pitch angle of $67.5^{\circ}$ " $\mathrm{CO}$ " with respect to the toroidal field. Note that particles approaching the detector with pitch angles of $\approx 85-90^{\circ}$ are blocked by the 
detector itself on the last orbit before entering the aperture, which was $1.5 \mathrm{~cm}$ below the box top (see Ref. 24).

\subsection{Geometrical resolution}

The geometrical pitch angle and energy resolving capability of this detector is described by Fig. 3. Tritons and protons approach the front "pinhole" aperture on helical orbits with gyroradii approximately $5 \mathrm{~cm}$ and typical pitch angles in the range $X-40^{\circ}-85^{\circ}$. Particles which pass through both the pinhole and the "slit" aperture are dispersed according to their pitch angle across one direction of the scintillator screen, as indicated. The same aperture pair also acts like a magnetic spectrometer and disperses particles according to their gyroradius along the orthogonal scintillator direction, also shown in Fig. 3.

A detector simulation code was written to clarify the geometrical resolution of this aperture/scintillator configuration. A set of helical orbits with a specified incident pitch arigle and gyroradius were constrained to pass through both the finite-sized rectangular front and rear apertures, and the resulting particle-impact positions on the scintillator were determined for various possible orbits allowed through the aperture pair. The actual angle of the aperture normal direction with respect to the toroidal field $\left(22.5^{\circ}\right)$ and the energy-attenuation effect of the foil (see below) were also included into the code.

Typical results for detector $\# 6$ are shown in Fig. 4 for 1 MeV tritons with an incident pitch angle of $X=60^{\circ}$. For this case the $0.2 \mathrm{~cm}$ wide front aperture sets a geometrical pitch angle resolution of about $\pm 3^{\circ}$ at the scintillator, and the $0.1 \mathrm{~cm}$ high front and rear apertures set an energy resolution of about a factor of two, i.e., the FWHM points of the $1 \mathrm{MeV}$ triton impact distribution coincide with the mean impact positions of 0.5 $\mathrm{MeV}$ and $2 \mathrm{MeV}$ tritons. These results are consistent with analytical estimates of resolution used earlier [25].

Therefore, this detector design had relatively good pitch angle resolution but poor energy resolution. The geometrical pitch angle resolution, in fact, was chosen to be about the same as the optical resolution. The relatively poor energy resolution was acceptable since the dominant energy spread was expected from the relatively uninteresting beam-target Doppler shifts. The aperture height also was limited by the 
need to eliminate the direct line-of-sight path through the aperture pair to the scintillator to prevent possible soft $x$-ray backgrounds.

\section{$2.4 \quad$ Foil}

Behind the slit aperture was a thin foil which blocked the plasma light and low energy plasma particles from entering the scintillator region. The foil was $3 \mu \mathrm{m}$ thick aluminum, which passed both $1 \mathrm{MeV}$ tritons (with about 25\% energy loss) and $3 \mathrm{MeV}$ protons (with $<3 \%$ energy loss), but which stopped the $0.8 \mathrm{MeV}{ }^{3} \mathrm{He}$ ions created in D-D reactions. This foil also set a low energy cut-off for triton or proton detection of about $300 \mathrm{keV}$.

The foil also acted as a scatterer of tritons or protons passing through them. The scattering angle for tritons in the $3 \mu \mathrm{m}$ aluminum foil is about 20 milliradian [26], which makes a slight contribution to the gyroradius resolution (see Sec. 3.2), but has negligible effect on the pitch angle resolution.

In order to check for possible differences between triton and proton loss, detectors \#9 had an $18 \mu \mathrm{m}$ aluminum foil which stopped the $1 \mathrm{MeV}$ tritons but passed (with $<20 \%$ energy loss) the $3 \mathrm{MeV}$ protons,. The results from this proton-only detector were at least qualitatively similar to the results for the triton/proton detectors \#6 and \#11. Detector \#4 for 15 MeV protons had a $275 \mu \mathrm{m}$ stainless steel "foil" and a very wide (non-energy resolving) aperture.

\subsection{Scintillator}

The scintillator, which was similar to that used in the prototype, consisted of a standard P11 ZnS(Ag) phosphor coating of thickness 10-15 $\mu \mathrm{m}$ (or 1-2 crystals) deep on top of a $1^{\prime \prime} \times 1^{\prime \prime} \times 0.1 \mathrm{~cm}$ quartz plate. A discussion of triton/proton detection with this scintillator can be found in Ref. 24.

A property of this P11 scintillator is that it emits less light per particle at temperatures above about $150^{\circ} \mathrm{C}$ than at lower temperatures ("quenching"). The temperatures inside the probe box were monitored with thermocouples and never exceeded $100^{\circ} \mathrm{C}$. for all data in this paper. 
It is plausible (but not experimentally demonstrated) that the light output of this scintillator should be proportional to the energy deposited in it by various $\mathrm{MeV}$ ions. Previous estimates based on this assumption [24] concluded that in this scintillator a 1 MeV triton creates about $1 / 3$ the light of a $3.5 \mathrm{MeV}$ alpha, and a $3 \mathrm{MeV}$ proton creates about 1/3 the light of a $1 \mathrm{MeV}$ triton (since the proton range in $\mathrm{ZnS}$ is about $100 \mu \mathrm{m}$ ). Thus about $75 \%$ of the light in this scintillator is expected to come from $1 \mathrm{MeV}$ tritons, and $25 \%$ from $3 \mathrm{MeV}$ protons, assuming equal numbers of tritons and protons incident on the detector. Based on the same assumption, the absolute calibration for tritons and protons was previously estimated from cross-calibrations with benchtop alpha particle sources $(241 \mathrm{Am}$ and $244(\mathrm{~m})$.

\subsection{Optics}

The scintillator image was transferred using a single $f / 2$ quartz lens $35 \mathrm{~cm}$ down a shaft, across a vacusum window, and into the coherent fiber optic bundle. The optiral characteristics of this system up to and including the video camera were calibrated before installation using a diffuse white light screen and a test-pattern grid at the scintillator plane. The spatial resolution was found through the "blurring" of a sharp black edge on a white background to be equivalent to about $\pm 0.2 \mathrm{~cm}$ at the scintillator plane, corresponding to a pitch angle resolution of about $\pm 5^{\circ}$ FWHM. The relative sensitivity across the scintillator plane gradually decreased by about $20 \%$ from center to edge, mainly due to "vignetting" in the single-lens optical system. Also present was some "blooming" in the video camera, particularly at high signal levels, which spread light from any bright object over adjacent parts of the image. This blooming effect was difficult to correct for precisely, but most of its effect was automatically included in the estimate of spatial resolution above.

The $8 \mathrm{~m}$ long plastic coherent fiber bundles had a 50x50 square array of $250 \mu \mathrm{m}$ fibers (Mitsubishi Super-Eska, numerical aperature 0.46). These fibers were aligned to about $t 1$ fiber, and were uniformly transmitting to within about $\pm 10 \%$ across the array. The four plastic fiber bundles of the poloidal detectors were viewed together in a square array through a $f / 2$ lens by the video camera. 


\subsection{Signal Detection}

The calculated detection efficiency (i.e., detected triton flux divided by global triton production) was typically $E \approx 10^{-8}$, so at a global triton source rate of $\approx 10^{16}$ tritons/sec the expected flux onto the scintillator was $=10^{8}$ tritons/sec. This rate was far too high for pulse counting, so only light flux measurements were made.

The light flux from the plastic fiber bundle was detected in one of two ways. Either a gated, intensified video camera was used to digitize the 2-D images vs. time with $32 \mathrm{msec}$ between video fieids, or else a linear array of eight $0.1 \mathrm{~cm}$ diameter plastic fibers was used to transfer the light from selected parts of the bundle to eight discrete photomultiplier tubes.

The video camera mode was used to evaluate the overall 2-D pattern of light at the scintillator and its slow-time variation. The camera (Xybion model ISG-03) had a controllable microchannel-plate gating time used to compensate for varying triton source levels; typically the gating time varied from $2 \mathrm{msec}$ at high triton levels to $16 \mathrm{msec}$ at low triton levels. The camera also had an internal automatic gain control of the internal microchannel-plate voltage, which was monitored by adding into its field of view a set of small but constant reference lights. The camera output was checked to linear with respect to light input, but had a $15 \%$ negative offset (which vyas corrected for in the analyzed data).

The photomultiplier (PM) tubes (Hamamatsu R762) were designed to look for fast fluctuations in the lost triton flux. The fibers leading to the PM tubes $(0.1 \mathrm{~cm}$ diameter Mitsubishi Eska-Extra) were proximity-coupled". to (i.e., directly touching) the 50x50 2-D bundle so as to cover 8 different pitch angles near the peak in the gyroradius coordinate (this obstructed the view of the bundle from the camera). The current from the PM tubes was monitored by the voltage drop across $100 \mathrm{kOhm}$ resistors by an amplifier with a bandwidth of $20 \mathrm{kHz}$. The voltage signals were digitized at rates of 5-100 $\times 10^{3}$ samples/sec by standard TFTR electronics.

\subsection{Data Systems}

The video camera data were transferred via a fiberoptic link to the TFTR control room, where it was digitized with a PC-based video 
frame-grabber board with $4 \mathrm{MB}$ memory (42 fields), and simultaneously archived in standard analog video-tape format. Customized image processing was available on the PC between shots. The video data was also sent to a VAX in compressed form ( 3 video lines $\times 3$ pixels $=$ one compressed pixely for further image processing.

The PM data were archived on the standard TFTR computer system. The timing of the PM data with respect to other fluctuation diagnostic data was accurate to within $10 \mu \mathrm{sec}$ when the fastest digitization rates were used.

\subsection{Backgrounds}

The main background in this system was a uniform illumination of the whole coherent bundle area with a light intensity proportional to the total neutron flux from TFTR, evidentally due to light created within the plastic fiber bundle by neutrons and/or gammas from the tokamak. This was checked by noting that the same background was seen on another plastic bundle of identical design which was run a similar distance inside the TFTR test cell $(\approx 3-4 \mathrm{~m})$, but which was not connected to any triton probe or other source of light. Note that in 1987 this background was lower since there was only $=1 \mathrm{~m}$ buidie length within the TFTR test cell.

The relative neutron/gamma background was reduced by adding an optical filter matched to the blue emission spectrum of $\mathrm{ZnS}(\mathrm{Ag})$ between the ends of the plastic bundle in the basement and the signal detectors (Kodak Wratten filter \#47). The remaining fiber bundle background was subtracted using an edge or a corner of the scintillator area not used for imaging the tritons.

The possible background caused by neutrons and/or gammas interacting with the scintillator itself [24] was apparently too small to observe in this system. There was also no perceptible background due to hard x-rays from runaway electrons, except after a major disruption when the scintillators were often brightly lit by a transient runaway electron burst.

Note that the soft $x$-ray background in the prototype was successfully eliminated in the present detector by adding $0.3 \mathrm{~cm}$ of stainless steel shielding above the scintillator, as shown in Fig. 2. The calculated reduction in the soft $x$-ray background was more than a factor of 100 , and 
in fact no perceptible soft $x$-ray background was observed with the present system.

\section{RESULTS FROM TFTR}

This section describes the experimental results obtained mainly from the \#6 triton/proton detector located near the bottom of the TFTR vacuum vessel. Particular attention is given to surveying a wide variety of TFTR conditions, and to comparing the video camera and PM signal-detection modes.

Wherever possible the results are compared to predictions from the PPPL single-particle first-orbit loss code ORBIT [17,25]. This code uses either modelled or experimentally derived triton source and plasma current profiles (from the FPPL analysis code SNAP), but ignores collisional effects, toroidal field ripple, and MHD instabilities (the effects of which are discussed briefly in Sec. 4.2).

For simplicity, the combined triton and proton flux measured by the detector \#6 will be called the "triton" flux, despite the fact that the signals measured by the this detector contain an estimated $25 \%$ contribution from $3 \mathrm{MeV}$ protons [24]. However, since the $3 \mathrm{MeV}$ protons and the $1 \mathrm{MeV}$ tritons have exactly the same gyroradii they also have exactly the same calculated first-orbit loss.

Sections 3.1-3.10 present a detailed comparison between the measurements and the first-orbit loss model for the bottom (\#6) detector for discharges with no strong MHD activity, Sec. 3.11 briefly describes observations of MHD-induced triton loss, and Sec. 3.12 summarizes observations from other detector locations.

\subsection{Typical 2-D Image}

A qualitative view of the triton imaging results is given in Fig. 5, which shows raw data from a typical 2-D image of light from the fiber bundle as measured by the video camera. The dark square near the borders of the plot corresponds to the boundary of the fiber bundle, the sides of 
which are about $10 \%$ larger than the image of the scintillator itself.

This data is from a standard $1.2 \mathrm{MA}, R=245 \mathrm{~cm}, a=80 \mathrm{~cm}$ discharge which had $14 \mathrm{MW}$ of neutral beam injection (NBI) for $0.7 \mathrm{sec}$, with an average neutron (and triton) rate of $6 \times 10^{15}$ neutrons/sec over the beam duration. For this figure the data are averaged over the whole discharge, which included about 20 video fields during NBI, each exposed for 4 msec. The shape of this pattern did nnt change significantly with time during this discharge.

The scales in Fig. 5 refer to the number of lines or pixels along each axis after a $3 \times 3$ compression; the actual video image of this detector covered $3 \times 41=123$ horizontal by $3 \times 40=120$ vertical lines. Note that this image is not corrected for the $15 \%$ negative offset of the video camera. Also, for this case, the blue filter was not inserted (see Sec. 2.9), which caused the fiber-bundle background to be somewhat larger than usual.

Note that the grid lines in this plot are oriented along the square scintillator boundaries, uncorrected for the pular geometry of the pitch angle vs. gyroradius coordinates implicit in the detector design (see Fig. 3). The horizontal axis in Fig. 5 (approximately) corresponds to the pitch angle coordinate of the scintillator, and the vertical (into the page) axis (approximately) corresponds to the gyroradius coordinate of the scintillator (this coordinate is roughly proportional to the square-root of the particle gyroradius [25]). These geometrical distortions are accounted for in the discussions of the gyroradius and pitch angle distributions below.

The qualitative result from this figure is clear and representative of all the data collected; namely, that there is a single, fairly well localized peak in the 2-D image from the scintillator, the position of which does not significantly change as the neutron rate varies with time during the discharge.

\subsection{Typical flux vs. Gyroroadius}

The first-orbit loss model predicts that tritons will be lost with very nearly their birth energy, since the time for one orbit $(<10 \mu \mathrm{sec})$ is much less than the collisional slowing down time $(\approx 0.5-1.0 \mathrm{sec})$. The triton birth energy is $1.0 \pm 0.2 \mathrm{MeV}$, the spread being due to the Doppler shift of beam-target reactions at a deuterium beam energy of $\approx 100 \mathrm{keV}$. Thus the 
gyroradius of lost tritons should be constant to within about $\pm 10 \%$.

Figure 6 represents a vertical "slice" through the peak signal region of this same 2-D image of Fig. 5 (lines \#14-21), showing the light flux along the "gyroradius" coordinate of the scintillator for the region within the fiber bundle. The distance along the scintillator is measured both in centimeters from the midpoint between the front and rear apertures (bottom scale), and in terms of the inferred triton energy (top scale). The inferred triton energy is calculated by the detector simulation code for an incident triton at a $60^{\circ}$ pitch angle (see Sec. 2.3), including the foil energy-attenuation effect.

The background curve in this figure is the intensity vs. line number from the same discharge evaluated at a position at the edge of the scintillator where the triton flux is expected to be negligible (lines \#30-34). Since the background from the plastic bundle itself is fairly uniform across the scintillator area, as was seen in cases with the bundle disconnected from a probe, this curve should approximately represent the background under the peak signal curve. Note that neither the signal nor the background curves are corrected for slight vignetting and blooming in the camera (see Sec. 2.6).

The peak of the net signal falls very near to the position expected tcr $1 \mathrm{MeV}$. This is observed on all discharges at this $\approx \mathbf{4 8} \mathrm{kG}$ toroidal field. The location of this peak was also shifted substantially with lowered toroidal field, just as expected from the gyroradius change for $1 \mathrm{MeV}$ tritons [25]. Note also that the peak of the expected $3 \mathrm{MeV}$ proton signal should be shifted only a small distance $(<0.1 \mathrm{~cm})$ from the triton peak due to the relatively lower foil attenuation effect.

The width of the observed signal appears to cover an inferred energy range $\approx 0.5-2.0 \mathrm{MeV}$. However, there are several sources of broadening expected for $1 \mathrm{MeV}$ triton first-orbit loss in this detector. First is the Doppler spread in triton energy of $\pm 20 \%$, or equivalently about $\pm 0.2 \mathrm{~cm}$ along the scintillator. Second is the geometrical spread expected due to the $0.1 \mathrm{~cm}$ front and rear aperture heights in this detector, which, as indicated by the shaded region in Fig. 6 , corresponds to a root-mean-square (r.m.s.) spread of about $\pm 0.25 \mathrm{~cm}$ (see Sec. 2.3). Third is the expected spread in this direction due to multiple scattering in the foil, which is about $0.1 \mathrm{~cm}$ for $1 \mathrm{MeV}$ tritons (see Sec 2.4).

Combined with these sources of broadening is the "optical resolution" 
of about $\pm 0.2 \mathrm{~cm}$ (see 5 ec. 2.6), indicated by the horizontal error bar on the data in Fig. 6. Together, these four factors create an expected width of about $\pm 0.4 \mathrm{~cm}$, which is quite close to the observed $\pm 0.4 \mathrm{~cm}$ FWHM signal width. There is also a systematic uncertainty of about $\pm 0.1 \mathrm{~cm}$ in the alignment between the experimental curves and the horizontal position scales (not shown).

The conclusion which can be drawn from this result is that the observed position of the peak of the signal along this gyroradius coordinate is consistent with the detection of the first-orbit loss $1 \mathrm{MeV}$ tritons (and $3 \mathrm{MeV}$ protons). Note, however, that the precise shape of the gyroradius distribution cannot be determined given the present system resolution; thus the potential presence of a small population of lower energy particles, e.g.. due to collisional loss effects, cannot be excluded by this measurement.

\subsection{Typical Flux vs. Pitch Angle}

The expected pitch angle dependence of first-orbit loss is sharply peaked around a "critical angle", which corresponds to the pitch angle of the fattest banana orbit for $1 \mathrm{MeV}$ tritons going through the given detector position [25]. A iypical example of this variation of the orbit trajectory with pitch angle is shown in Fig. 7 (a). At the critical pitch angle of $\approx 55^{\circ}$, the trajectory or "sightline" of the orbit passes nearest to the high triton source rate region near the plasma center, and so the escaping flux should be largest at this pitch angle. Figure 7 is drawn for $1=1.6 \mathrm{MA}$ plasma current, but $₹$ similar picture applies over the normal current range of TFTR 0.8 - 2.0 MA.

Figure $B$ represents a horizontal "slice" through the peak signal region of the same 2-D image of Fig. 5 (lines \#15-22), showing the light flux vs. the pitch angle coordinate at the scintillator for the region within the fiber bundle. The distance across the scintillator is measured in centimeters from its geometrical center, which is aligned to correspond to a pitch angle of $67.5^{\circ}$ with respect to the "co" direction of plasma current. The pitch-angle scale at the top is calculated from the detector simulation code for this horizontal slice position, i.e., including the slightly polar geometry and the effect of the foil on the particle gyroradius. The approximate backgrourd curve is estimated from a set of lines at the edge 
of the scintillator where no tritons are expected (lines \#28-32). Again, these curves are not explicity corrected for the slight vignetting and blooming effects in the camera.

The peak of the observed signal in Fig. 8 falls at a pitch angle of about $55^{\circ}$ with respect to the "co" toroidal direction. This is quite near to the expected critical pitch angle of $59^{\circ}$ calculated by the first-orbit loss code at 1.2 MA for $1 \mathrm{MeV}$ tritons at this detector, as shown by the solid region in this figure. The calculated critical pitch angle is constant to within about $\pm 2^{\circ}$ for reasonable variations in the assumed plasma current profile, here taken to be parabolic-cubed with a Shafranov shift of $0.2 a$ (for comparison, with zero Shafranov shift, the critical pitch angle shifts from $59^{\circ}$ to $57^{\circ}$ ). There is also a systematic uncertainty in the optical alignment of about $\pm 0.1 \mathrm{~cm}$, or $\pm 3^{\circ}$ (near $60^{\circ}$ ), not shown in the figure.

Note that this idealized first-orbit loss curve in Fig. 8 does not include the $\pm 3^{\circ}$ geometrical pitch angle broadening expected due to the 0.2 $\mathrm{cm}$ width of the front aperture (see Sec. 2.3), nor the broadening due to the $\pm 20 \%$ Doppler energy spread in triton energy, or the effect of averaging over the lines used to obtain the experimental curve, the latter two of which each produce about a $\pm 1^{\circ}$ spread in the predicted peak. Thus, the expected broadening of the model curve is about $\pm 4^{\circ}$ around the idealized shape.

Thus, the combined widths of the ideaiized model curve (about $\pm 5^{\circ}$ ), the expected instrumental broadening $\left( \pm 4^{\circ}\right)$, and the "optical resolution" $\left( \pm 5^{\circ}\right)$ leads to an expected signal width of about $\pm 8^{\circ}$, which at present we interpret to be in reasonable agreement with the observed width of $\pm 10^{\circ}$. Unfortunately, the precise shape of the pitch angle distribution, and the expected sharp drop in flux below the critical angle, cannot be identified given the present system resolution.

The conclusion which can be drawn from this section is that the position and width of the light signal vs. pitch angle is consistent with the expected first-orbit loss. Small variations in the peak of the pitch angle distribution with varying plasma current are discussed in Sec. 3.7.

\subsection{Typical Flux vs. Time}

Figure 9 shows the light flux vs. time for the same discharge of Secs. 3.1-3.3, as monitored by the video camera and averaged over the center of 
the peak region (horizontal lines \#14-21, vertical lines \#15-22). The time dependence of the peak light flux approximately follows the neutron signal monitored by the standard epithermal neutron detectors [27]. The background, which was evaluated at the corner of the scintiliator, also follows the neutron flux as expected.

This rough linearity between the lost triton flux and the neutron flux is typical of all discharges without strong MHD activity. This behavior is also qualitatively consistent with the expected prompt first-orbit loss, since the loss time is only $\approx 10 \mu$ sec. However, the triton source and curpent profiles can also affect the ratio of lost tritons to neutrons in a given discharge at a fixed plasma current (see Sec. 3.9). These and other time-dependent effects are more conveniently monitored using the photomultiplier detection mode, as described next.

\subsection{Typical Photomultiplier Signals}

For most of the 1988 run the triton light signals from the \#6 detector were monitored with the eight channel photomultiplier (PM) array instead of the video camera made used for Secs. 3.1-3.4 above. This was done by proximity-coupling eight plastic fibers directly to the coherent fiber bundle. These 8 fibers were distributed along the approximate pitch angle direction of the scintillator near the peak of the signal in the gyroradius direction, either using 0.1 or $0.2 \mathrm{~cm}$ spacing between fibers. Note that for all PM data discussed here, the blue filter was used to reduce the background light level from the plastic fiber bundle (see Sec. 2.9).

Typical pitch-angle distributions inferred from these fibers are

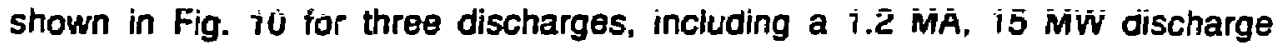
similar to that used with the video camera mode in Secs. 3.1-3.4, and also a 2.0 MA (17.5 MW) and a 1.0 MA (17.5 MW) discharge. For this figure the signals were integrated over 0.5 sec during NBI injection when there was no strong MHD activity, and normalized by the averaged neutron flux during this time.

These pitch angle distributions all have a peak near $58^{\circ}$ similar to the distribution obtained using the video camera monitor in Fig. 7. The widths of these distributions appear to be about $\pm\left(5^{\circ}-10^{\circ}\right)$, i.e., somewhat narrower than the $\pm 10^{\circ}$ inferred from the video camera. This was expected due to absence of the camera lens and some slight camera 
"blooming" in the video mode. However, in this PM mode the discrete fiber spacing of about $5^{\circ}$ limited the pitch angle resolution. There was also an exira background in the PM mode from neutron/gamma pickup in the PM tube fibers or in the PM tubes themselves; this was checked by decoupling these fibers from the coherent bundle and still observing half the background noted in Fig. 10.

The time dependences of two of these PM channels for the 1.2 MA. discharge are shown in Fig. 11, here smoothed with a $10 \mathrm{msec}$ averaging interval. Both the peak signal channel at about $58^{\circ}$ and the background channel at about $83^{\circ}$ have a time dependence similar to that of the neutron signal, as expected from the video camera result of Sec. 3.4 .

The conclusion which can be drawn from this section is that the results from PM tubes are consistent with those from the the video camera with respect to the pitch angle and time dependences of the signals, as illustrated by a typical 1.2 MA discharge.

\subsection{Flux vs. Plasma Current}

As discussed in Sec. 1.1, the first-orbit confinement of $\mathrm{MeV}$ ions should be determined mainly by the plasma current, and should improve dramatically over the range $=0.5-2.0 \mathrm{MA}$. An illustration of this effect is given in Fig. $7(\mathrm{~b})$, where at $0.8 \mathrm{MA}$ the trajectories of $1 \mathrm{MeV}$ tritons from the plasma center can reach the bottom detector, while at 1.6 MA only the trajectories from about $r / a>0.3$ can do so.

The typical variation with plasma current of the overall 2-D pattern of triton flux at the scintillator is shown in Fig. 12. This set of data comes from a plasma current scan from 0.9-1.4 MA, and includes the same 1.2 MA discharge used in Secs. 3.1-3.4. The NBI power for this data set was 12-14 MW, the plasma size was $R=245 \mathrm{~cm}, a=80 \mathrm{~cm}$, and the toroidal field was $B=46 \mathrm{kG}$.

Each phots of Fig. 12 shows raw data from a single video field exposed for $4 \mathrm{msec}$ and chosen at a time during NBI when the instantaneous neutron flux was $(4.5 \pm 0.5) \times 10^{15}$ neutrons/sec. The light intensity is displayed here using a linear false-color scale (difficult to reproduce in black and white). The scintillator area itself is indicated by the black corners of the 1.2 MA case, and the data retain the small negative offset of the video camera. 
The qualitative result is clear and typical of all results under MHD-quiescent conditions. Namely, the shape of the gyroradius and pitch angle distributions of the light flux are approximately independent of plasma current, and the total light flux decreases substantially with increasing plasma current at a fixed neutron source rate. The independence of the gyroradius distribution on plasma current implies that the escaping triton energy distribution is peaked at about $1 \mathrm{MeV}$, independent of current as expected for first-orbit loss. The approximate independence of the pitch angle distribution on plasma current follows from the approximate invariance of the critical pitch angle on plasma current (see Sec. 3.7).

A quantitiative analysis of the total escaping triton flux on plasma current for this same current scan over 0.9-1.4 MA is shown in Fig. 13. Here the light flux for each of 15 shots was measured from single video frames again exposed for $4 \mathrm{msec}$ when the neutron flux was $(4.5 \pm$ $0.5) \times 10^{15} \mathrm{n} / \mathrm{sec}$. Points representing both the peak signal region (defined as in Sec. 3.4) and average signals (over the scintillator area) are shown, with the background subtracted using a region inside the scintillator area but outside the peak region. Esoth measures of escaping triton flux per neutron, i.e., the relative triton loss fraction, decrease about a factor of three between 0.9 and $1.4 \mathrm{MA}$.

A second test of the current dependence was made in the PM mode. with results as shown in Fig. 14. This data set came from a different current scan monitored over a somewhat larger current range of $0.8-1.6$ MA, with NBI power of 7-28 MW, plasma size $R=245 \mathrm{~cm}, a=80 \mathrm{~cm}$, and toroidal field $B=48 \mathrm{kG}$. This figure includes 73 high-power, neutral-beam heated discharges, where each point represents the net triton flux seen by the fibers iur one discharge (i.e., the sum of the three PM signals which were within the peak in the pitch angle distribution, minus the background level measured at the edge of the scintillator). normalized by the total neutron flux during the same integration time. The integration times during NBI varied from $0.08-1.5$ sec for these shots.

The resulting normalized triton flux, or relative triton loss fraction, decreases by about a factor of six between 0.8 and $1.6 \mathrm{MA}$ and by about a factor of three between 0.9 MA and 1.4 MA similar to Fig. 12. Note that the background as monitored at the edge of the scintillator was small and constant for this data set, i.e., between 0.1 and 0.2 on this scale for all currents. A similar plasma current dependence can also be seen in the pitch-angle resolved PM data for three different currents in Fig. 10. The 
results obtained using a single fiber near the peak behave similarly to the summed triton signal in Fig. 14, since the pitch angle distribution did not vary substantially over this current range.

This observed decrease of relative triton loss fraction with plasma current roughly agrees with the simplified first-orbit loss model prediction, as indicated by the theoretical curve normalized to the data of Fig. 14 at $0.8 \mathrm{MA}$. This model curve represents the total expected $1 \mathrm{MeV}$ triton loss integrated over the pitch angle range $=50^{\circ} .80^{\circ}$ for this detector, calculated assuming a constant triton source profile (parabolic to the sixth), a constant plasma current profile (parabolic to the third). and no Shafranov shift in the current distribution.

However, this simplified model used in Fig. 14 is not entirely consistent with the data, since this model predicts a factor of 11 reduction in triton flux per neutron over this same current range, and not the factor of six seen. Part of this discrepency may simply be a systematic underestimate of the background (and so overestimate of the signals at high current), which could be due to a lower optical efficiency for the fiber at the edge of the scintillator compared to the fiber near the center. Another apparent inconsistency with the first-orbit loss model is the apparent scatter of about $\pm 25 \%$ r.m.s. in the data at fixed plasma current. Attempts to explain these effects in terms of systematic variations in the plasma current and/or triton source profiles are discussed in Secs. 3.9 .

The conclusion from this section is that the lost triton flux decreases with increasing plasma current, at least qualitatively, as expected from the first-orbit loss model. If the poloidal distribution of this loss were invariant with current, the observed decrease would imply that that the triton loss fraction at $1.6 \mathrm{MA}$ must be $<16 \%$, since the loss at $0.8 \mathrm{MA}$ can be at most $100 \%$, which would imply good "single-particle" alpha confinement for reactor-grade machines. However, the actual poloidal distribution of triton loss (particularly near the outer midplane) remains to be determined.

\subsection{Effect of Plasma Current on Pitch Angle Distribution}

Figure 15 shows video camera data for the pitch angle distributions of two of the discharges previously shown in the color photos of Fig. 12. 
These raw data plots are averaged over the same gyroradius range used for Fig. 8 , but are displayed before $3 \times 3$ compression for better pitch angle resolution. There is a shift of the peak toward lower pitch angles in the higher current case which was seen consistentally throughout the run.

Figure 16 shows this variation in the peak pitch angle for the set of shots used for Fig. 13. Experimentally, there is an $\approx 5^{\circ}$ decrease in the pitch angle of the peak over the curreni range 0.9-1.4 MA, while the first-orbit loss models predicts a $4^{0.70}$ decrease. The model curves are shown for both a simplified parabolic-cubed, unshifted current profile, and also for the actual Shafranov-shifted current profile (as inferred from neoclassical resistivity for individual TFTR shots over this current range), indicating that this decrease in pitch angle is not sensitive to the assumed current profile.

Thus, the relative change of the pitch angle distribution with plasma current agrees fairly well with the first-orbit model, although the absolute pitch angles of the points are consistently about $5^{\circ} .7^{\circ}$ lower in pitch angle than the Shafranov-shifted calculation. This discrepancy is apparently larger than the estimated $\pm 3^{\circ}$ uncertainty in the absolute calibration of pitch angle at the scintillator (see Sec. 2.3).

\subsection{Variation of Flux with Neutron Source Strength}

As already seen implicitly in the relative triton loss fraction plot of Fig. 14, the lost triton flux increases approximately linearly with the global neutron (triton) creation rate at a given plasma current. For that data set the observed $\pm 25 \%$ r.m.s. scatter in the relative triton loss was not correlated with the neutron source strength or the integration time. The approximate linearity between neutron source strength and escaping triton flux is also implicit in their similar time dependences, as shown previously in Figs. 9 and 11.

This dependence is made explicitly using a data set for 1.6 MA plasmas in Fig. 17. This data includes 169 (randomly chosen) 1.6 MA, 10-30 MW, $R=245 \pm 2 \mathrm{~cm}, \mathrm{~B} \approx 49 \pm 3 \mathrm{kG} \mathrm{NBI}$ shots from a month-long TFTR run period where the set-up of the PM fibers was held constant, including 114 discharges without perceptible MHD activity (" $x "$ "). This data set includes none of the discharges discussed so far.

The relative triton loss fraction was obtained using the 
time-averaged signal from the fiber nearest the peak flux $\left(\approx 58^{\circ}\right)$, after subtracting out the signal from a fiber near the edge of the scintillator $\left(83^{\circ}\right)$, and normalizing the result by the integrated neutron flux during the time. The integration time during NBI varied from $1.6 \mathrm{sec}$ (covering the entire NBI phase) to $0.08 \mathrm{sec}$. Note that the neutron-normalized background level itself was approximately constant at $0.7 \pm 0.1$ in these units for all shots, i.e., about $30 \%$ of the net signal level.

The result from Fig. 17 is that the normalized triton flux is approximately constant over a factor of 15 in the neutron source strengh $(0.2-3.0) \times 10^{16} \mathrm{n} / \mathrm{s}$, with the best linear fit to the data having only a $25 \%$ increase over this range. Thus, this result is, at least qualitatively, consistent with the first-orbit loss model, which predicts a corstant triton loss fraction if the triton source and plasma current profiles are constant.

However, there is also an unexpected $\pm 25 \%$ r.m.s. scatter around the mean for the discharges without MHD activity, with the highest and lowest points differing by a factor of $2-3$ at a neutron rate of $\approx 3.0 \times 10^{16} \mathrm{n} / \mathrm{sec}$. Note that most of this scatter is not due to shot-to-shot variations in the plasma or to statistical fluctuations, since the normalized triton loss for a consecutive sequence of discharges under constant plasma conditions typically varies by $<10 \%$ r.m.s.

There are several potential instrumental reasons for this scatter. One is day-to-day uncertainty in the relative calibration of the neutron detectors used for the normalization; however, with the best available neutron calibrations (as applied to Fig. 17), this uncertainty is about $\pm 5 \%$ [27]. Another is the day-to-day calibration of the PM tubes for the triton measurement, which was not monitored explicitly, but which is probably less than $\pm 10 \%$ (since the voltage and configuration were not changed during the run, and since there is no systematic trend in the scatter vs. discharge number). A third possible effect comes from the typical $\pm 10-20 \%$ variation in the triton to neutron ratio vs. time during a discharge, as shown in Fig. 11; however, sub-sets of this data constrained to constant integration times had a similar scatter.

The conclusion which can be drawn from this section is that the approximate constancy of the normalized triton flux is consistent with the simple first-orbit loss picture, but there may also be some unexplained scatter in the data. Attempts to understand such variation in terms of triton source and plasma current profiles are described in the next section. 


\subsection{Profile Etfects}

The lost triton flux at a given plasma current can be affected by both the triton source profile and the plasma current profile. Generally, the lost triton flux is higher when the line integral of the triton source along its orbit is larger; this happens if the source strength becomes higher along the orbit, or if changes in the current profile shift the orbit to a region of higher source strength.

\subsubsection{Carculated Profile Effects}

The calculated source-profile effects for $1.6 \mathrm{MA}$ discharges are summarized by one curve in Fig. 18, where the calculated triton detection efficiency " $\varepsilon$ " for the present detector is plotted vs. the source profile shape factor $S\left(r_{\min }\right) / S(0)$, where $\varepsilon$ is the calculated triton flux through the aperture pair divided by the global neutron (triton) rate. $S\left(r_{\min }\right)$ the local source rate at the distance o: closest approach of the orbit to the plasma center, $r_{m i n}$, which is independent of the assumed triton source profile, and $S(0)$ is the triton source rate at the plasma center. This figure is calculated for conditions similar to that for the data of Fig. 17. i.e., a plasma size of $R=245 \mathrm{~cm}, a=80 \mathrm{~cm}$, and a fixed current profile taken from one of the shots in Fig. 17 (\#37084). The triton pitch angle at the detector was assumed to be $60^{\circ}$, for which case $r_{\min } / a=0.38$ (see Fig. 7 ). The triton source profile shape was varied from parabolic to the $1^{\text {st }}$ power to parabolic to the $11^{\text {th }}$ power, i.e., $S(r) \alpha S(0)\left(1-[r / a]^{2}\right) P$, where the exponent $P=1-11$ is indicated by the number along the curve. The implication of this result is that a variation of the triton source profile shape from $S\left(r_{\min }\right) / S(0)=0.2$ to 0.4 can change the expected triton flux by about a factor of two.

Also, in Fig. 18 are two curves showing the calculated effect of the assumed plasma-current-profiie shape on $\varepsilon$, taking into account the variation in $r_{\min }$ with current-profile shape given fixed source profiles. In both cases the current-profile shape was assumed to be parabolic to a 
power varying from $p=1-7$, with the power indicated by the number along the curves. The source-profile shape was assumed to be parabolic to the $4^{\text {th }}$ for the curve on the right and parabolic to the $10^{\text {th }}$ for the curve on the left. The implication of these curves is that the current profile can also affect the expected $\varepsilon$ by up to a factor of two, especially given the possibility of strongly driven or non-equilibrium current profiles in TFTR.

Thus, the observed variation of a factor of 2-3 between the lowest and highest points in the experimental data of Fig. 17 is not inconsistent with possible profile effects. The next part of this section attempts to find a correlation between experimental profile shapes and this scatter in the 1.6 MA ojta of Fig. 17.

\subsubsection{Comparison with the Data}

One way to check for profile effects is to use the shot-specific triton source and plasma current profiles (including the Shafranov shift) as generated by the PPPL analysis code SNAP in the triton detection efficiency code ORBIT, and compare the computed efficiency with the observed efficiency for each discharge. A small but random set of 12 shots of the 114 shots without MHD of Fig. 17 had appropriate SNAP analyses available for comparison, with results as shown in Fig. 19.

The result is that the computed efficiency varied over the range $\varepsilon(1.5-2.1) \times 10^{-8}$ for the shots with no MHD effects, apparently uncorrelated with the observed variation of a factor of two in the relative triton loss fraction, whereas a linear relation between the relative triton loss and the calculated efficiency would be expected from profile effects. A similar result applies to 12 other shots with MHD effects (see Sec. 3.11). Note that the analyzed discharges without MHD fall in the range $S\left(r_{\min }\right) / S(0) \approx 0.3$, i.e., the typical experimental source profile was approximately parabolic to the $7^{\text {th }}-9^{\text {th }}$ (including beam-target and beam-beam sources).

Another way to look for possible profile effects is to plot the experimental triton efficiencies of Fig. 17 against some more directly measured experimental paramaters related to profiles. This is done in Figs. 20 and 21.

In Fig. 20 the triton data is plotted against the electron density 
peaking factor $n_{e}(0) /<n_{\theta}>$, as determined from the multichannel interferometer system [28] at the time of maximum stored energy for each discharge. The region below $n_{e}(0) /<n_{e}>\approx 2$ is conventionally considered the "L-mode" regime of degraded plasma confinement, and that above $\approx 2$ is considered the "supershot" regime of improved plasma confinement. The supershot regime has more rentrally peaked neutron source strengtins but bioader current profiles than the L-mode regime (according to SNAP). trends which tend to cancel each other out with respect to triton detection efficiency. In the data, there seems to be a small systematic trend for the lost triton detection efficiency to increase with the density peakedness parameter for discharges whout MHD, as it the curcent bro."...'ng offect dominated the source peaking effect.

In Fig. 21 the same triton data of Fig. 17 is plotted against the parameter $\Lambda\left(=l_{i} / 2+\left[\beta_{p \|}+B_{p \perp}\right] / 2\right)$ for each discharge, as dervied from magnetic measurements at the peak of the stored energy. This $\Lambda$ is a (nearly linear) measure of the Shafranov shift of the plasma center with respect to the outermost magnetic flux surface. Evidentally there is a weak increase in the normalized triton flux with increased Shafranov shift (for discharges without MHD). Orbit code calculations for $1.6 \mathrm{MA}$ discharges of this type show a $30 \%$ increase in the expected detection efficiency with increased Shafranov shift over this range (up to 0.2a). Incidentally, it is not surprising that the triton efficiency increases with neutron rate, density profile peaking, and $\Lambda$, since these three parameters are correlated with each other (and with neutral beam power) in this database.

A similar attempt to find a correlation between the normalized triton flux and the plasma density itself showed only a slight increase of flux with density, which might be associated with broader triton source profiles at higher density. No correlation was found between normalized triton flux and central electron temperature.

A more successful attempt to demonstrate profile effects was made using the current scan data of Fig. 14, in which there was observed to be a less-than-expected decrease in the triton flux whan a simplified fixed-profile efficiency calculation was used. When SNAP-generated profiles from shots in this sequence were used instead, the calculated detection efficiency ratio between 1.0 MA and 1.6 MA was $2 \pm 0.5: 1$, whereas the calculated efficiency ratio for the fixed profiles used in Fig. 14 was 
6:1. Therefore, the experimental ratio of $2.5 \pm 0.5: 1$ between $1.0 \mathrm{MA}$ and 1.7 MA discharge is better fit by the SNAP-generated profiles, which incorporated the significantly broader source and current profiles calculated for the higher current cases.

The tentative corclusion is that any profile effects on triton loss at a fixed plasma current are too subtle to observe directly in the database at 1.6 MA, although the rather gross profile effects associated with differing currents can apparently be observed. Further understanding of these triton profile effects will probably require direct measurements of the $2.5 \mathrm{MeV}$ neutron source and plasma current profiles (which were not routinely available during the 1988 run).

\subsubsection{Other Possible Profile Effects}

We conclude this section by mentioning two other possible profile effects. The first concerns the influence of the NBI direction, and the second concerns the effect of time-varying plasma current.

The effect of NBI direction is shown in Fig. 22 for a set of 1.1 MA data with either all co-(i.e., along 1) or all counter beam injection (note that the data for Fig. 17 was mainly balanced injection). The consistent result of these and other cases was that the normalized triton flux was about $40 \%$ higher for counter-injection than for co-injection, with balanced injection being nearer to the levels for co-injection (a similar effect can also be seen in discharges with time-dependent changes in NBI direction). However, neither the calculated triton source profiles nor the calculated plasma current profiles (including beam-beam reactions and beam-driven currents, respectively) were significantly different between these co- and counter-injection cases, as analyzed by SNAP and TRANSP.

An alternative explanation for the co-/counter- effect is that the triton birth distribution became significantly anisotropic in velocity space due to the to the Doppler shift of the beam-beam and beam-target reactions, causing the sightline-integrated triton loss to dependent on the sign of this anisotropy (the present orbit efficiency code assumes an isotropic source). The expected effect of this anisotropy would be in the same direction as observed; namely, to increase the lost flux when the rotation produced more reactions in the counter direction near the plasma center. 
A second effect is illustrated in Fig. 23, which shows the influence of a time-dependent plasma current "ramp" on the normalized triton flux. In this case the normalized flux decreases by only about $30 \%$ when the total plasma current is raised from 1.1 to $1.7 \mathrm{MA}$, in contrast to the factor of 3 decrease expected from the data of Fig. 14 and the factor of 6 decrease expected from calculations assuming normal plasma current and source profiles. Apparently, the time-dependent profiles are much broader in this ramp case, leading to a relatively high triton loss. A simulation of possible "surface" current profiles was made with the ORBIT code, which showed that if the additional $600 \mathrm{kA}$ of plasma current was localized near $r / a=0.8$ then the expected triton flux decreases by only a factor of 2 . However, further interpretation of these discharges is complicated by MHD aciivity during the current ramp, which appears in this case at 3.7-3.8 sec.

\subsection{Plasma Position Effects}

The effect of plasma position on the escaping triton flux is shown in Fig. 24. For these discharges, all at 1.4 MA, the normalized triton flux (summed over pitch angle) is shown for plasma major-radius positions of $R$ $=238 \mathrm{~cm}, 245 \mathrm{~cm}$ (the standard position) and $252 \mathrm{~cm}$. The striking observation is that the triton loss fraction at $R=238 \mathrm{~cm}$ is larger by a factor of about $\times 2-3$ than that at $R=245 \mathrm{~cm}$ or $252 \mathrm{~cm}$. This trend has consistently been observed on several different occasions during the 1988 run.

Application of the ORBIT code to these different plasma positions predicts that the triton flux should be approximately independent of plasma major radius, since for a given pitch angle the triton orbit tends to move along with the plasma flux surfaces. In fact, the code predicts a $30 \%$ decrease in flux at $R=238 \mathrm{~cm}$ compared to $R=245 \mathrm{~cm}$ when using SNAP-generated source and plasma current profiles for discharges in this data set.

One possible resolution of this discrepancy is that the finite spacing between PM fibers (see Fig. 10) allowed small shifts in the pitch angle distribution to affect the total signal seen by the fibers (i.e., the peak signal at $R=245 \mathrm{~cm}$ may have occured between two adjacent fibers, resulting in an underestimate of the net signal). However, the orbit code predicts that the peak of the pitch angle distribution should shift from $59^{\circ}$ 
at $R=245 \mathrm{~cm}$ to $56^{\circ}$ at $R=238 \mathrm{~cm}$ and to $60^{\circ}$ at $A=252 \mathrm{~cm}$, which seems too small a shift to cause the observed variation in net signal. In fact, examination of the pitch angle distributions vs. A shows, if anything, a slight increase in the peak pitch angle at $R=238 \mathrm{~cm}$, in contradiction to the orbit code.

Therefore, the R-dependence of the lost triton flux shown in Fig. 24 apparently cannot presently be understood on the basis of the first-orbit loss model. Possible explanations could involve unexpectedly broad profiles in these plasmas (compared with those calculated by SNAP), or MHD activity (however, low-frequency MHD was not observed on these $A=$ $238 \mathrm{~cm}$ triton signals). Such effects are suggested by the fact that the triton loss fraction at $R=238 \mathrm{~cm}$ for these discharges was almost linearly correlated with the neutron rate, suggesting some systematic anomaly with increasing stored energy in these cases.

Note that the vertical position was not varied significantly during the 1988 run; however, earlier results showed no signifcant changf in the escaping triton flux with vertical position changes of a few $\ldots n$, in agreement with the orbit code.

\subsection{MHD Effects}

Large coherent MHD activity was often observed to increase the triton loss, particularly in discharges at higher currents, as shown in the $1.6 \mathrm{MA}$ database plot of Figs. $17[16,29]$. At this current about $30 \%$ of the high power discharges (>10 MW) had perceptible coherent oscillations on the triton $P M$ signals near the peak pitchangle region of $X=50-70^{\circ}$ (with no oscillations on the background channels at other pitch angles). Sometimes these oscillations persisted through most of the NBI phase and resulted in increased average loss, while at other times the MHD activity was either too weak or too brief to affect the integrated loss. The triton loss fraction for discharges without MHD in the 1.6 MA database was $1.8 \pm 0.4$ (in the units of Fig. 17), while for shots with MHD it was $2.5 \pm 1$, indicating a marginally significant increase overall with MHD.

Although a detailed discussion of this phenomenon is beyond the scope of the present paper, one example of this MHD effect is shown in Figs. 25 and 26. In this particular 1.6 MA discharge the net triton flux integrated over the whole discharge is increased about a factor of two above the level 
of a similar discharge without MHD. In both figures the triton data comes from the same PM channel used for Fig. 17, which had a $20 \mathrm{kHz}$ analog bandwidth and a sampling rate of $5 \mathrm{kHz}$ in these cases.

When the MHD frequency was below about $10 \mathrm{kHz}$ (as in Fig. 26), the triton flux was clearly modulated with the MHD oscillations, with a maximum triton flux up to $\times 10$ above the triton loss level in the absence of MHD. In many cases the MHD mode frequency decreased to near zero (mode "locking"), during which time the triton flux remained anomalously high, as during the period from 4.25-4.35 sec in Fig. 25. When the Mi:ID frequency was above about $10 \mathrm{kHz}$ the modulations in the triton flux were not clearly visible (due to the analog bandwidth of the PM circuit), but the triton flux was also increased above the level without MHD. Thus, some of the unusually high points "without MHD" in Fig. 17 may have been cases with either locked or high frequency modes which were not perceptible as modulations on the triton signal.

This effect of MHD activity on triton loss has been observed for several different types of coherent MHD modes, sawtooth crashes and fishbone-type oscillations. Further analyses of these phenomena is in progress.

\subsection{Other Poloidal Detectors}

There were several other $\mathrm{MeV}$ ion detectors installed during the 1988 TFTR run, as shown in Fig. 1. Poloidal array detectors \# 9 and \#11 had relativley small signal levels due to their lower optical coupling efficiency $(=1 / 3$ that of \#6), but were monitored regularly with the video camera in parallel with detector \#6. Detector \#4 was designed for $15 \mathrm{MeV}$ protons and saw no signals, since there were few appropriate $\mathrm{D} .{ }^{3} \mathrm{He}$ discharges with ICRF heating. The four detectors of the toroidal array also had small signals due to inefficient optical coupling and were not monitored regularly.

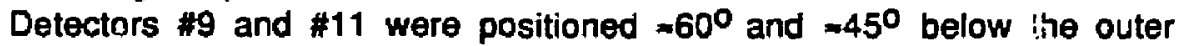
equatorial plane, respectively. They had an aperture/scintillator geometry very similar to that of \#6, except that the plane of the scintillator, apertures, and box tops was aligned parallel to the local flux surfaces (see Fig. 1). Detector \#11 had a $3 \mu \mathrm{m}$ aluminum foil which passed tritons and protons like detector \#6, but detector \#9 had an $18 \mu \mathrm{m}$ aluminum foil 
which passed only protons.

A typical pitch angle distribution from detector \#11 is shown in Fig. 27 for a 1.2 MA discharge similar to that used for $\# 6$ in Fig. 8 (i.e., $R=245$ $\mathrm{cm}, \mathrm{a}=80 \mathrm{~cm}, 16 \mathrm{MW} \mathrm{NBI}$ ). The distribution is peaked at about $47^{\circ}$, i.e., at a significantly lower angle than for $\# 6$. However, the first-orbit loss code predicts that the critical angle should be about $42^{\circ}$ for this detector location, and so this result is roughly consistent with the code (given the $\pm 5^{\circ}$ systematic uncertainty in the pitch angle alignment). Other signatures of first-orbit loss seen in this detector are: (a) the gyroradius distribution for detector is consistent with $1 \mathrm{MeV}$ tritons (cf. Fig. 6); (b) the time dependence was similar to that of the neutron flux (cf. Fig. 9); and (c) the flux decreased with plasma current (cf. Fig. 13).

For the proton-only detector \#9 the pitch angle, energy (i.e., gyroradius), time and plasma current dependence of the signals had the same qualitative features as those seen in the triton/proton detectors \#6 and \#11. However, the peak in the pitch angle distribution was consistently in the $60^{\circ}-65^{\circ}$ range, as shown in Fig. 28, which is significantly larger than the first-orbit code prediction of a $53^{\circ}$ critical angle at this poloidal location. At present there is no explanation for this apparent discrepency.

The ratio of the peak signal levels between detectors \#6 and \#11 was $=1 \pm 0.5$ at $1.2 \mathrm{MA}$ (after correction for the optical efficiency). This agrees fairly well with the expected ratio 1.3:1 obtained from the first-orbit code for these two detector locations. However, the ratio of the signals between detector \#6 and detector \#9 was also about 1:1 (after correction for optical efficiency), which, after also correcting for the expected ratio of light flux for protons in \#9 to tritons+protons in \#6 (see Sec. 2.5), implies that the proton flux in detector $\# 9$ is about 4 times the triton flux in detector \#6. This disagrees with the orbit code, which predicts the flux at detector position \#9 should be about 0.85 that of detector \#6. Therefore, either there are losses other than first-orbit losses to detector $\# 9$, or the expected triton/proton light output ratio of the scintillator is incorrect (this has not yet been measured experimentally).

The behavior of these signals with varying major radius was also unexpected. The normalized flux in detector \#9 decreased by about $\times 2$ between $\mathbf{A}=245 \mathrm{~cm}$ and $238 \mathrm{~cm}$, in contrast to the flux in detector \#6 which increased by about $x 2$ for the same change (Sec. 3.10). Also, the triton loss fraction in detector \#11 decreased more than a factor of four 
for the smaller plasmas; however, it is possible that the triton orbits were intercepted by a toroidal array detector downstream from \#11 (see Fig. 1).

Improved measurements of the triton flux with poloidal angle are needed to resclve these points. Measurements near to the midplane of TFTR are scheduled for 1990.

\section{CONCLUSIONS}

This paper described measurements of the loss of alpha-like MeV ions into detectors located at the bottom of the TFTR vessel. The results were compared with the expected first-orbit loss due to the large banana widths of these ions at the plasma currents of 0.8-1.6 MA.

\subsection{Summary of Results}

For escaping tritons measured $=90^{\circ}$ below the outer midplane, in discharges with normal minor radius without strong MHD activity:

1) the pitch angle and energy distributions of lost tritons were consistent with the expected first-orbit loss, i.e., the loss was mainly at a pitch angle near $60^{\circ}$, corresponding to the fattest banana orbit, and at $=1 \mathrm{MeV}$, corresponding to prompt loss at the birth energy;

2) the time dependence of the triton loss followed the time dependence of the neutron (triton) source rate, as expected for prompt loss;

3) the decrease in the lost triton fraction (i.e., lost triton flux normalized by the global triton araation rate) with increased plasma current was approximately consistent with the first-orbit model;

4) a observed shift in peak of the pitch angle distribution with changing plasma current was approximately consistent with first-orbit mode! predictions, although the absolute value of the pitch angles was somewhat different from that calculated; 
5) the average triton loss fraction at a fixed plasma current was approximately constant over the whole range of TFTR D-D reaction rates, as expected from the first-orbit loss model for constant triton source and plasma current profiles;

6) the observed scatter of about $\pm 25 \%$ r.m.s. in the normalized lost triton flux at 1.6 MA could not be directly correlated with changes in plasma source or current profile shapes;

7) the triton loss fraction increased significantly for small major radius plasmas in a way not expected from the first-orbit loss model;

8) in discharges with strong MHD activity, the average triton loss fraction increased up to 2-3 times the level without MHD, these increases being modulated in phase with the MHD mode for $f<10 \mathrm{kHz}$;

9) the triton and proton loss observed in detectors located $45^{\circ}$ and $60^{\circ}$ below the outer midplane was qualitatively similar to that at $90^{\circ}$, as expected for first-orbit loss; however, the pitch angle distributions at these locations did not quite agree with the first-orbit code, and the estimated flux to the $60^{\circ}$ proton-only detector was larger than expected.

\subsection{Discussion of Results}

The general conclusion of these results is that the loss of alpha-like $\mathrm{MeV}$ ions as observed at the bottom of TFTR appears to be consistent with the expected first-orbit loss. Of course, it is not surprising that MeV ions are lost this way. The most interesting aspects of these results are the observation of some apparently "anomalous" (non-first-orbit loss) effects, and also the apparent absence of some other potentially important non-first-orbit effects.

The most striking anomaly was the increased triton loss associated with strong coherent MHD (Secs. 3.8 and 3.11). There are several possible causes for this: a) modification of the triton source profile by the MHD, such that regions of increased source periodically cross the triton trajectory, b) magnetic "ripple" induced loss of previously trapped tritons 
due to the low-n perturbations [11], or c) leakage of previously confined passing tritons across the passing-trapped boundary due to non-axisymmetric "kicks" of the orbits by the MHD [31]. Further clarification will require improved source profile measurements and more detailed theoretical modelling. Note that large MHD activity is expected in reactor-grade plasmas near the high- $\beta$ limit and/or if collective alpha instabilities are excited [32].

Another major anomaly was the factor-of-two larger than expected triton loss observed for small major radius plasmas in the bottom detector (Sec. 3.10), correlated with an unexpectedly small triton loss for the same plasmas in the $45^{\circ}$ detector (Sec. 3.12). One plausible explanation could be that the triton source or plasma current profiles are broader than presently calculated by SNAP for these smaller plasmas. Another possible explanation could be the presence of (unobserved) high frequency MHD activity in the smaller, lower $q(a)$ plasmas.

A third but less certain anomaly concerns the unexpectedly large flux and the somewhat unexpected pitch angle distribution in the proton-only detector $=60^{\circ}$ below the outer equator. A possible cause for these effects is additional proton loss due to the TF field ripple. The theoreticallypredicted stochastic TF ripple-diffusion process [6-8] causes the banana tips of confined trapped ions to randomly walk vertically, resulting in a loss which tends strongly toward the outer midplane region [9].

Preliminary results from the simplified RIPLOS code [11] predict a negligible stochastic TF ripple loss for normal TFTR plasmas; however, there are subtleties in more sophisticated treatments which require extremely large Monte-Carlo computations. Unfortunately, there are presently no quantitative predictions for the poloidal and pitch angle distributions of this loss in TFTR, so it is presently difficult to exclude possible TF ripple-induced loss in the present results, particularly since global TF ripple losses tend to be relatively prompt ( $=10 \mathrm{msec}$ loss time), and to decrease with increasing plasma current similarly to prompt losses. Note that "superbanana" loss due to ions trapped in the TF-ripple wells cannot be observed in the present detectors, since this loss occurs at pitch angles near $90^{\circ}$ which are blocked in the present detector design.

Another theoretically predicted classical effect is the loss of partially thermalized $\mathrm{MeV}$ ions due to classical pitch angle scattering of confined ions born near the passing-trapped boundary [3,33]. An early analytic estimate of this effect for alpha particles in a 1 MA TFTR plasma 
predicted a cumulative collisional loss fraction of $\approx 1 \%$ for TFTR-like plasmas [3], which was approximately $10 \%$ of the first-orbit loss in that calculation. Since the predicted energy of these losses was mostly within about $20 \%$ of the birth energy, it would be difficult to exclude the presence of such an effect in the gyroradius distribution of Fig. 6, or by the absence of delayed triton loss after NBI (since $20 \%$ triton energy loss occurs over about $0.2 \mathrm{sec}$, and since triton scattering is much slower than alpha scattering). More careful measurements are needed to check this effect, perhaps with a detector having better energy resolution.

A third potentially important effect which was not observed in these experiments, was a large anomalous radial triton diffusion such as is observed for thermal ions in tokamaks. For the present experiment such diffusion might be observable if confined passing tritons near the plasma center moved radially outward, since they should eventually cross the passing-trapped boundary (if their magnetic moment is conserved) and so should be lost on the fattest banana orbits visible from our detectors. It is most likely that this apparently low MeV ion diffusion is due to the "orbit averaging" effect of large ion orbits in the presence of small-scale turbulence [11], as discussed elsewhere [34]. Other evidence for a relatively low diffusion rate for fast ions has been obtained for $100 \mathrm{keV}$ NBI ions on TFTR [35], and ICRF minority ions on JET [36].

In summary, the confinement of alpha-like MeV ions as measured with these escaping triton detectors in TFTR appears to be consistent with the simple first-orbit loss model, which implies that alpha confinement ought to be excellent in reactor-grade plasmas, assuming collective effects remain negligible. However, observations of enhanced triton loss during strong MHD activity, and the possiblity of enhanced losses near the (presently undiagnosed) outer midplane warrant further experimental and theoretical investigations.

\subsection{Potential Improvements}

The present results are also limited in several respects which suggest further improvements in experimental technique:

1) Measurements should be made near the outer equatorial plane where the main TF ripple loss is expected [9] and where anomalous loss due to slow 
diffusion of confined orbits might also occur. Anomalous triton loss at the midplane could explain the anomalously low triton burnup often seen on TFTR [16]. A new outer midplane triton detector was recently installed on TFTA for this reason [30].

2) These present measurements were all made with detectors located about $2 \mathrm{~cm}$ behind the limiter, which does not affect the collection of first-orbit loss (since these orbits move outward radially more than $2 \mathrm{~cm}$ in the toroidal distance between the limiter and the detector), but which may miss any slow radial diffusion of trapped tritons to the poloidal region of the jetectors. The new midplane triton probe will be radially movable in order to explore this effect.

3) Measurements of the triton and proton light outputs from these scintillators are needed in order to obtain an absolute calibration of these detectors. An absolute scintillator calibration is presently being made using a scintillator located inside a $D-D$ generator.

4) The spatial resolution of the present detector and camera imaging system is only marginaily able to determine the shapes of the triton pitch angle and energy distributions; for example, the expected sharp cutoff at the critical pitch angle is not seen in the data of Fig. 8. Therefore, effects such as the source profile dependence of the pitch angle distribution and the possible presence of low energy triton components cannot be seen with the present system. Replacement of the plastic coherent fiber bundles with quartz should increase the signal levels by $x 4$ and so allow improved detector spatial resolution through reduced aperture sizes.

5) Independent measurements of the plasma current and triton birth profiles are needed to resolve the effect of these profiles on triton loss. Input from the new multichannel neutron collimator should be very useful in this regard. 


\section{ACKNOWLEDGMENTS:}

Thanks go to many people who contributed to this project. Help with the hardware came from G. Barnes, T. Deverell, D. Johnson D. Manos, J. Montague, K. Owens, R. Palladino, E. Tolnas, and M. Ulrickson. Help with the data analysis codes came from C.W. Barnes, J. Felt, W. Heidbrink, J. Roberts, S. Scott, and M. Zarnstorff. The magnetics data used for Fig. 21 came from M.E. Bell, and the magnetic fluctuation data for Figs. 25 and 26 came from E.D. Fredrickson. Additionally, we thank R. Duvall, H. Mynick, D. Sigmar and R. White for discussions of the theory, and H. Furth, R. Goldston, L. Johnson, D. Meade and K. Young for their support for this project.

This work was supported by U.S. Department of Energy Contract \#DE-AE02-76-CHO3073. 


\section{REFERENCES}

1) KOLESNICHENKO, YU. I., Nucl. Fusion 20, 727 (1980).

2) ANDERSON, D., HAMNEN, H., and LISAK, M., Next European Torus Report \#91, EUR-FU/80/88-9".

3) HIVELY, L.M. and MILEY, G.H., Nucl. Fusion 20, 969 (1980).

4) BITTONI, E., HAEGI, M., SANTINI, F., and SEGRE, S.E., Nucl. Fusion 22. 1675 (1982).

5) ANDERSON, D. and BATISTONI, P., Nucl. Fusion 28, 2151 (1988).

6) GOLDSTON, R., WHITE, R.B., and BOOZER, A.H., Phys. Rev. Lett. 47, 647 (1983).

7) TANI, K., TAKIZUKA, T., AZUMI, M., and KISHIMOTO, H., Nucl. Fusion 23, 657 (1983).

8) BELIKOV, V.S., KOLESNICHENKO, YU. I., and YAVORSKIJ, V.A., Fusion Technology 15 (1989) 1365.

9) GOLOBOROD'KO, V. Ya., and YAVORSKIJ, V.A., Nucl. Fusion 29, 1025 (1989).

10) MYNICK, H.E. and DUVALL, R.E., Phys. Fluids B 1, 750 (1989).

11) WHITE, R.B. and MYNICK, H.E., Phys. Fluids B 1, 980 (1989).

12) HEIDBRINK, W.W., CHRIEN, R.E., and STRACHAN, J.D., Nucl. Fusion 23 , 917 (1983).

13) HEIDBRINK, W.W., HAY, R., and STRACHAN, J.D., Phys. Rev. Lett. 53, 1905 (1984). 
14) BATISTONI, P., B!TTONI, E., and HAEGI, M., Nucl. Fus. 29, 673 (1989).

15) CONAOY, S., JARVIS, O.N., SADLER, G., and HUXTABLE, G.B., Nucl. Fusion 28, 2127 (1989).

16) STRACHAN, J.D., ZWEBEN, S.J., BARNES, C.W., BOSH, H.-S., FURTH, H.W., et al, Proc. $12^{\text {th }}$ Int'l. Conference on Plasma Physics and Controlled Nuclear Fusion Research, Nice (1988), IAEA-CN-50/A-IV-5, Vol. 1, p 257.

17) CHRIEN, R.E., KAITA, R., STRACHAN, J.D., Nucl. Fus. 23,1399 (1983).

18) HEIDBRINK, W.W., LOVBERG, J., and STRACHAN, J.D., and BELL, R.E., Nucl. Fusion 27, 129 (1987).

19) BOSCH, H.-S., and SCHUMACHER, U., Proc. 12 th European Conf. on Controlled Fusion and Plasma Heating, Schliersee, 1986, Vol. 10C, Part II. European Physical Society, 124.

20) MUAPHY, T.D. and STRACHAN, J.D., Nucl. Fusion 25, 383 (1985).

21) SiTRACHAN, J.D., Nucl. Fusion 29, 163 (1989).

22) MARTIN, G., JARVIS, O.N. and KALLNE, J., Physica Scripta T16, $17^{1}$ (1987).

23) ZWEBEN, S.J., Physica Scripta, T16, 119 (1987).

24) ZWEBEN, S.J., Rov. Sci. Inst. 60, 576 (1989).

25) ZWEBEN, S.J., Nucl. Fusion 29, 825 (1989).

26) SEGRE, E., Nuclei and Particles, Benjamin Press, p. 46.

27) HENDEL, H.W., PALLADINO. R., et al, Princeton Plasma Physics Laboratory Report PPPL-2679 (19::0).

28) PARK, $H$. et al, Princeton Plasma Physics Laboratory Report 
PPPL-2651 (†989).

29) ZWEBEN, S.J., STRACHAN, J.O., BOIVIN, R., BARNES, C.W., CAVALLO, A., et al, $16^{\text {th }}$ European Conference on Contrclled Fusion and Plasma Heating, Venice 1988.

30) BOIVIN, R.L., DUVALL, R., MYNICK, H., WHITE, R., and ZWEBEN, S.J., Bull. Am Phys. Soc. 34, 2014 (1989).

31) DUVALL, R.E., Ph.D. Thesis, Princeton University (1990).

32) SIGMAR, D.J., MIT Plasma Fusion Center Report \#PFC/JA8958 (1989).

33) CATTO, P.B., Phys. Rev. Lett. 60, 1954 (1988).

34) ZWEBEN, S.J., Physics of Fluids $B$ (to be published)

35) RADEZTSKY, R.H., SCOTT, S.D., KAITA, R., GOLDSTON, and R.J., HAMMETT, G.W., et al.,15 th European Conf. on Controlled Fusion and Plasma Heating, Dubrovnik, 1988, European Physical Society, Vol. 1, p. 79-82.

36) START, D.F.H., BHATNAGAR, V.P., BOYD, D.A., et al, Proc. IAEA Tech. Committee Meeting on Alpha Particles in Fusion Devices, Kiev (1989) [Fusion Technology, 1990].

37) J.D. STRACHAN, private communication (1990) 


\section{FIGURE CAPTIONS}

Fig. 1 Location of escaping triton/proton detectors in TFTR during the 1988 run period. Most of the data was obtained from the \#6 detector located at $\mathbf{R}=259 \mathrm{~cm}$, approximately $90^{\circ}$ below the outer equator. Some data was also obtained from the $\# 9$ and $\# 11$ detectors located $-60^{\circ}$ and $=45^{\circ}$ below the outer equator, respectively.

Fig. 2 Mechanical design of detector \#6. The top of this light-tight detector box is located $2 \mathrm{~cm}$ behind the ICRF limiter radius of $99 \mathrm{~cm}$. The front aperture was $0.1 \mathrm{~cm}$ high vertically and $0.2 \mathrm{~cm}$ wide, the rear aperture was $0.1 \mathrm{~cm}$ high and $1.5 \mathrm{~cm}$ wide, the aperture spacing was $1 \mathrm{~cm}$, the distance between the aperture center and the scintillator center was $1.5 \mathrm{~cm}$, and the vertical distance between the aperture centers and the scintillator plane was $0.2 \mathrm{~cm}$. A thin foil is also located behind the rear aperture to block plasma light. Detectors \#9 and \#11 were similar in design, except that the box tops were tilted to be parallel to the local flux surfaces.

Fig. 3 Functional design of the triton detectors. Tritons with an energy of $1 \mathrm{MeV}$ and a gyroradjus $\neq 5 \mathrm{~cm}$ are incident on helical orbits. These orbits must pass through the front aperture "pinhole" and rear aperture "slit" in order to reach the scintillator. Tritons are dispersed according to their pitch angles and gyroradii in the directions shown.

Fig. 4 Detector simulation code results for $1 \mathrm{MeV}$ tritons incident at $60^{\circ}$ pitch angle on the \#6 detector. Each circle represents the scintillator impact position of one possible trajectory through the finite-sized aperture pair. The pitch angle resolution is mainly set by the front aperture wisth, and the gyroradius (energy) resolution is mainly set by the aperture heights.

Fig. 5 Raw data of a typical 2-D pattern of light intensity from detector \#6, showing the triton impact to be concentrated in one region of the scintillator plane. The boundary of the fiber bundle is shown by the 
darkened rectangle (the boundary of the scintillator is slightly smaller). This data was integrated over the whole NBI phase of a 1.2 MA discharge (\#35113).

Fig. 6 Gyroradius distribution of the light intensity across the peak of the signal in Fig. 5. The peak signal came at an inferred triton energy of 1 MeV, with a FWHM ranging from $0.5-2 \mathrm{MeV}$. This broadening is expected due to the finite aperture size and optical resolution.

Fig. 7 Calculated trajectories of some typical $1 \mathrm{MeV}$ triton orbits which pass through detector \#6. In (a) the orbits for three pitch angles are shown, all for a plasma current of 1.6 MA and for a fixed plasma current profile. The fattest banana orbit occurs at a "critical angle" of $=55^{\circ}$ when the orbit most closely approaches the plasma center and the largest triton flux is expected. In (b) the orbits for $65^{\circ}$ pitch angles are shown for 1.6 and $0.8 \mathrm{MA}$, illustrating the expected decrease in flux with increased current.

Fig. 8 Pitch angle distribution of the light intensity across the peak in the signal of Fig. 5. The maximum signal came at an inferred pitch angle of $\approx 55^{\circ}$, which is close to the peak in the calculated curve at $59^{\circ}$ indicated by the shaded region. The broadening of the signal curve with respect to the simplified model curve is expected from the detector's geometrical resolution (error bar on the shaded curve) and the optical resolution (error bar on the data). There is also an $\pm 3^{\circ}$ uncertainty in the pitch angle alignment of the detector (not shown).

Fig. 9 Time dependence of the peak signal from the same discharge used for Fig. 5, as digitized from the video camera data with a time-resolution of 32 msec. The net triton signal follows the time dependence of the 2.5 MeV neutron flux, as expected for first-orbit loss. The background signal due to neutron/gamma-induced light in the fiber bundle was monitored using a corner of the scintillator not used for triton detection.

Fig. 10 Pitch angle dependence of the light flux as determined by the photomultiplier tube (PM) monitoring system. The results for a $1.2 \mathrm{MA}$ discharge (\#37054) are similar to the video camera results of Fig. 8 . Other discharges at 1.0 MA (\$37063) and $2.0 \mathrm{MA} \mathrm{(37068)}$ have similar pitch 
angle dependences.

Fig. 11 Time dependence of the light flux from a single PM fiber positioned near the peak of the 2-D light distribution (580 pitch angle) for the $1.2 \mathrm{MA}$ discharge of Fig. 10. The result is similar to that for the video camera monitor in Fig. 9.

Fig. 12 Overall pattern of the escaping triton flux vs. plasma current. Each of these pictures shows raw data from a single video frame exposed for $4 \mathrm{msec}$ when the neutron flux was $(4.5 \pm 0.5) \times 10^{15}$ neutrons/sec. The position of peak intensity was roughly invariant at $=60^{\circ}$ pitch angle and 1 $\mathrm{MeV}$ inferred triton energy while the intensity decreased with increasing plasma current, as expected for first-orbit loss. The boundary of the scintillator itself is indicated by the four corners in the $1.2 \mathrm{MA}$ case.

Fig. 13 Triton loss fraction vs. plasma current for the data set used in part for Fig. 12 (\$35106-35123). The peak triton flux falls by about $\times 3$ over this current range.

Fig. 14 Triton loss fraction vs. plasma current for a series of discharges monitored using the PM detectors (*35205-35453). The measured flux decreases approximately as expected from a simplified first-orbit loss model in which the profiles are kept constant. The background level, as monitored by another fiber, was constant at between 0.1 and 0.2 on this scale, and has already been subtracted out.

Fig. 15 Examples of pitch angle distributions for two of the discharges used in the current scan of Fig. 14. The signal peak is shifted to a lower pitch angle at the higher plasma current.

Fig. 16 Peak pitch angle of the measured triton flux vs. plasma current for the data set of Fig. 14. The trend to lower pitch angles at higher current is predicted by the first-orbit code. The model including the Shafranov shift in the profiles is probably the more realistic one.

Fig. 17 Triton loss fraction vs. neutron source strength for a large set of 1.6 MA discharges with nearly constant major radius $R=245 \pm 2 \mathrm{~cm}$ (within the range \#35365-37944). The average triton loss fraction does not vary 
significantly with neutron source strength for discharges without MHD activity, as expected for first-orbit loss with constant profiles. The discharges with MHD often have increased triton loss.

Fig. 18 Calculated effects of the triton source and plasma current profiles on the triton detection efficiency for detector \#6 at 1.6 MA. The curves represents models with parabolic profiles to a power indicated by the number on the line. Experimental source profiles inferred from the SNAP code are typically fit by by a parabolic to the $7-9^{\text {th }}$ power. Given these sensitivities, variations of a factor-of-two in detection efficiency over the discharges in the 1.6 MA database are to be expected.

Fig. 19 Comparison between observed triton loss fractions and calculated detection efficiency using SNAP plasma current and source profiles for some shots in the 1.6 MA database. The observed variations apparently cannot be identified with the calculated profile effects.

Fig. 20 Variation of the normalized triton flux with the electron density profile peaking factor. For discharges without MHD there is a slight trend toward increased triton loss at high values of this parameter, possibly associated with the broadened current profiles. The discharges are again 1.6 $\mathrm{MA}$ at $\mathrm{R}=245 \pm 2 \mathrm{~cm}$.

Fig. 21 Variation of the normalized triton flux with magnetically inferred $\Lambda$ for the same $1.6 \mathrm{MA}, \mathrm{R}=245 \pm 2 \mathrm{~cm}$ data set in Fig. 17. For discharges without MHD activity there is a slight tendency toward increased triton loss at high $\Lambda$, possibly associated with increased Shafranov shift.

Fig. 22 Variation of the normalized triton flux with co-/counter- balance for a set of uni-directional NBI discharges at $1.1 \mathrm{MA}$. There is a significantly higher flux for counter-injection cases; however, this trend cannot be explained based on calculated profile effects.

Fig. 23 Variation of the triton loss fraction with time during a current-ramp discharge. The observed flux falls by only $30 \%$ between 1.1 and 1.7 MA, compared to the expected fall of $\times 3$ observed for normal steady-state discharges, suggesting a large profile broadening effect during this ramp. 
Fig. 24 Effect of plasma major radius on triton loss fraction for a set of 1.4 MA discharges (within \#36365-37994). There is an unexpected increase in flux for the small major radius plasmas.

Fig. 25 Effect of strong MHD activity on the time-dependence of the lost triton flux for one of the discharges in Fig. 17 (\$37956). The average flux increases by about a factor of two above the level for discharges without MHD.

Fig. 26 Fast-timescale behavior of the triton flux for the discharge shown in Fig. 25. The triton flux is clearly modulated at the same frequency as the MHD oscillations observed by a magnetic pickup coil at the plasma edge.

Fig. 27 Typical pitch angle dependence of the triton flux into detector $\# 11$ at $\approx 45^{\circ}$ below the outer midplane (\#35323). The pitch angle peak is shifted with respect to that in detector \#6 (Fig. 8), bui roughly agrees with the calculated first-orbit loss indicated by the shaded region. There is a $\pm 5^{\circ}$ uncertainty in the pitch angle calibration of the experimental data (not shown).

Fig. 28 Typical pitch angle dependence of the triton flux into the proton-only detector $\# 9$ at $=60^{\circ}$ below the outer midplane ( $\# 35323$ ). There appears to be a significant difference between the observed and calculated pitch angle distributions for this detector, although the qualitative features of the proton loss are similar to the triton loss seen in detectors \#6 and \#11. 


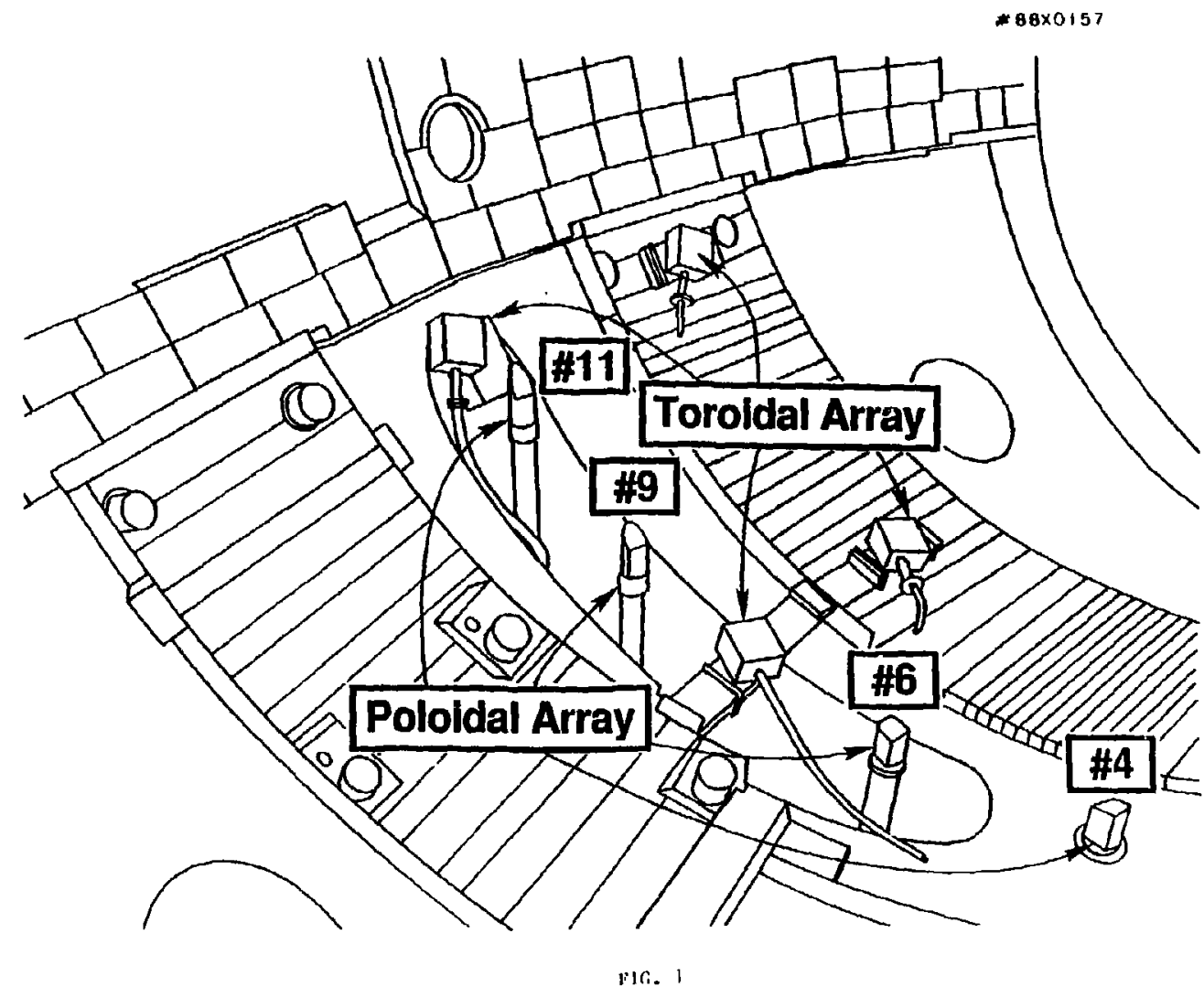




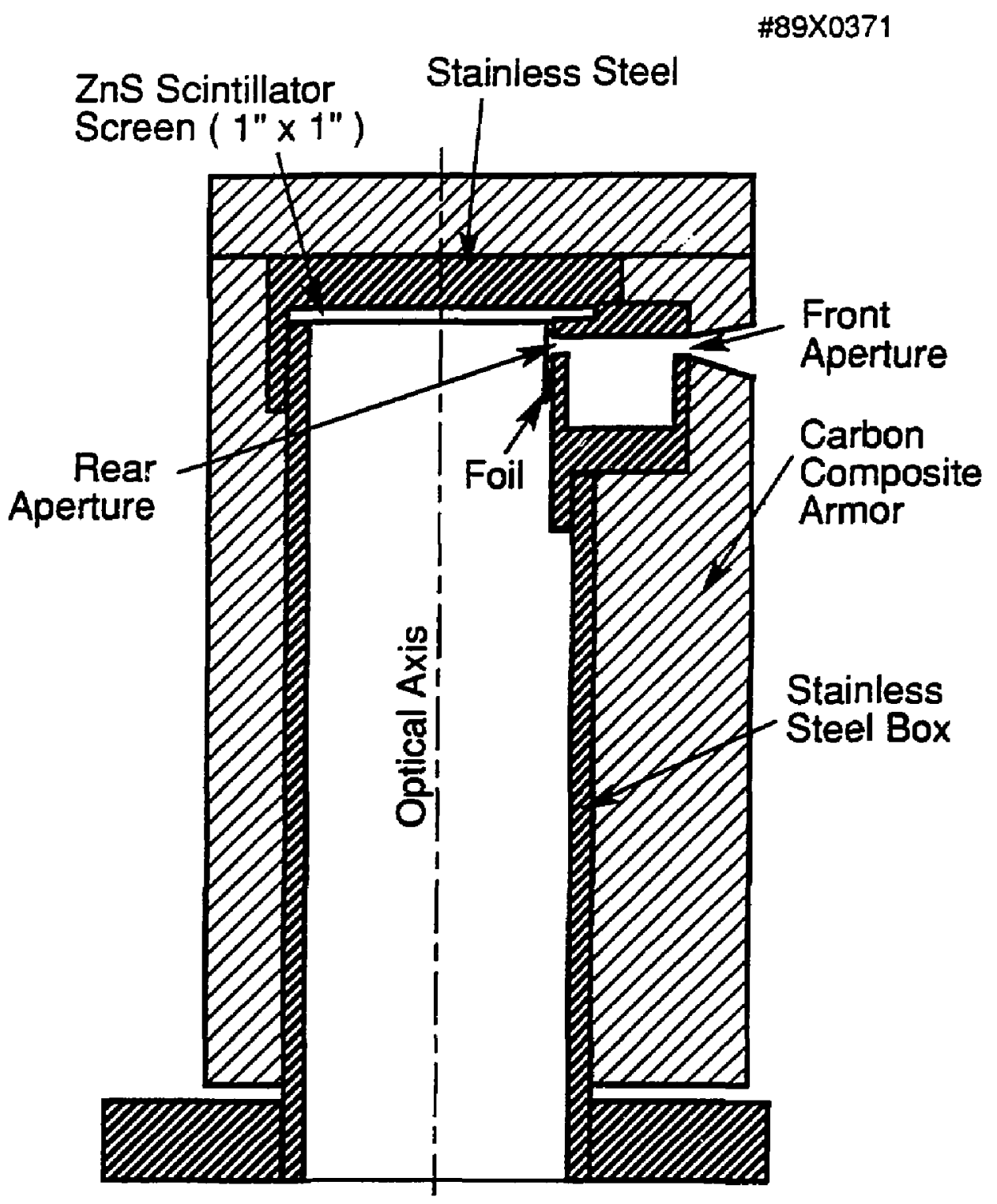




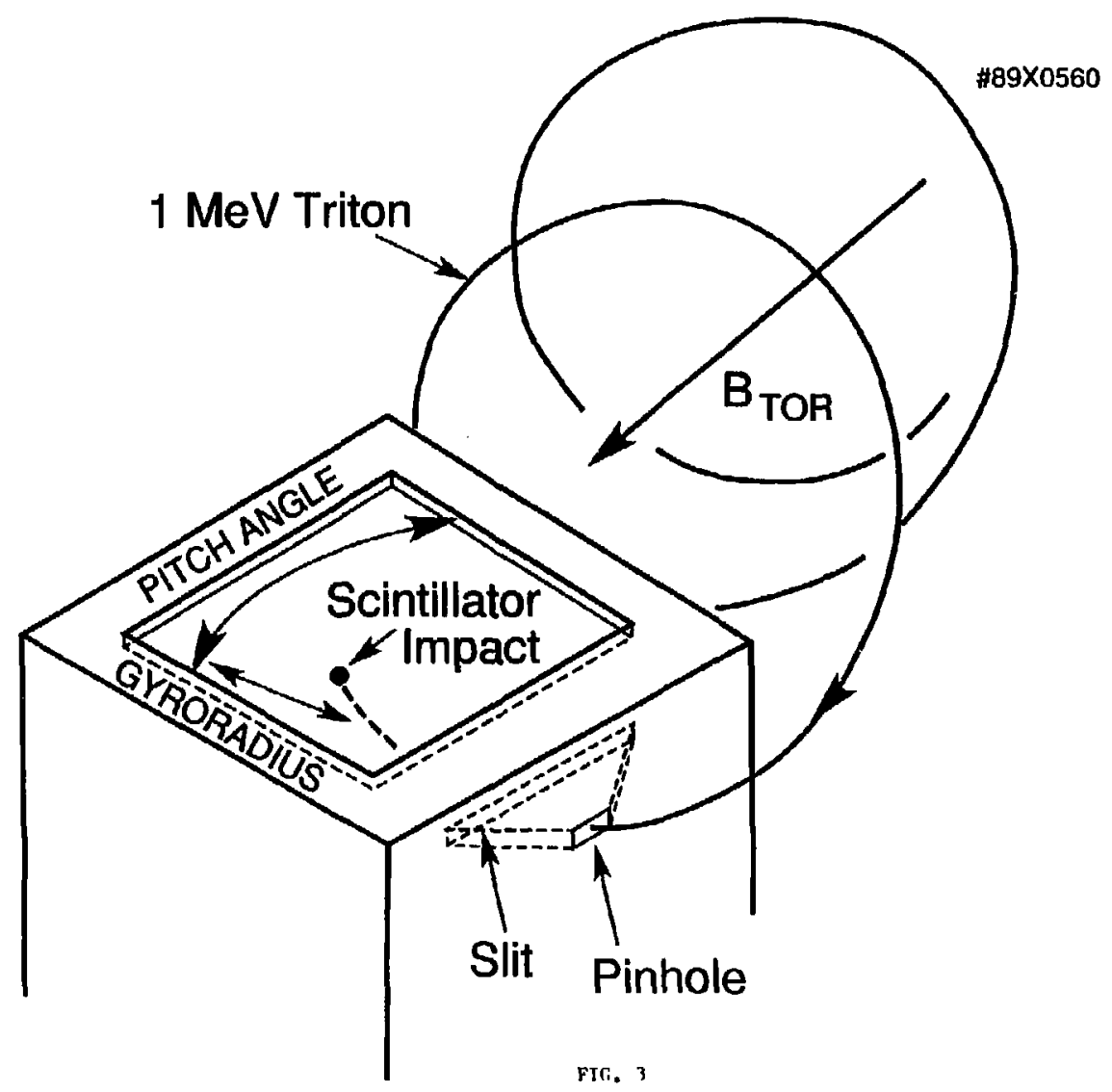


*89X0557

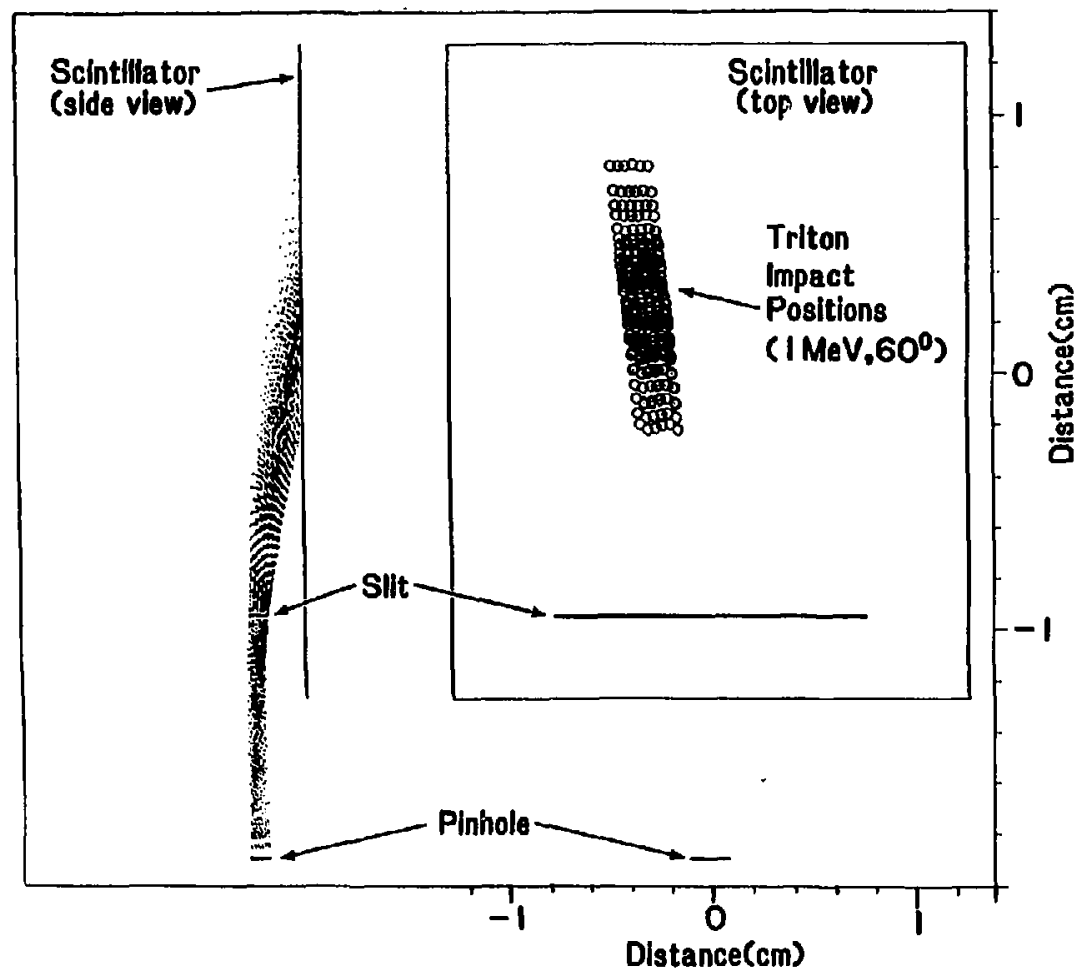

FIG. 4 


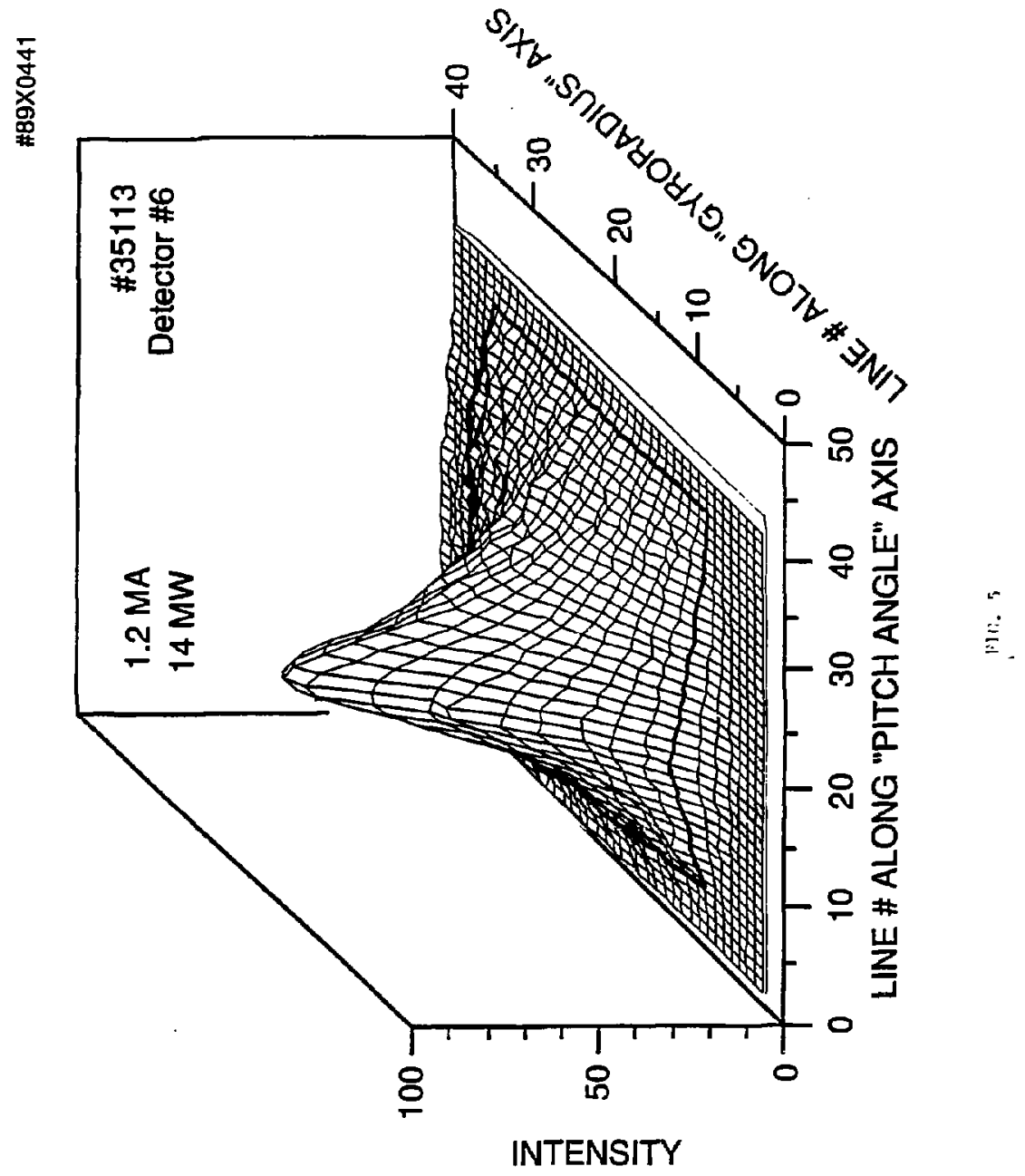




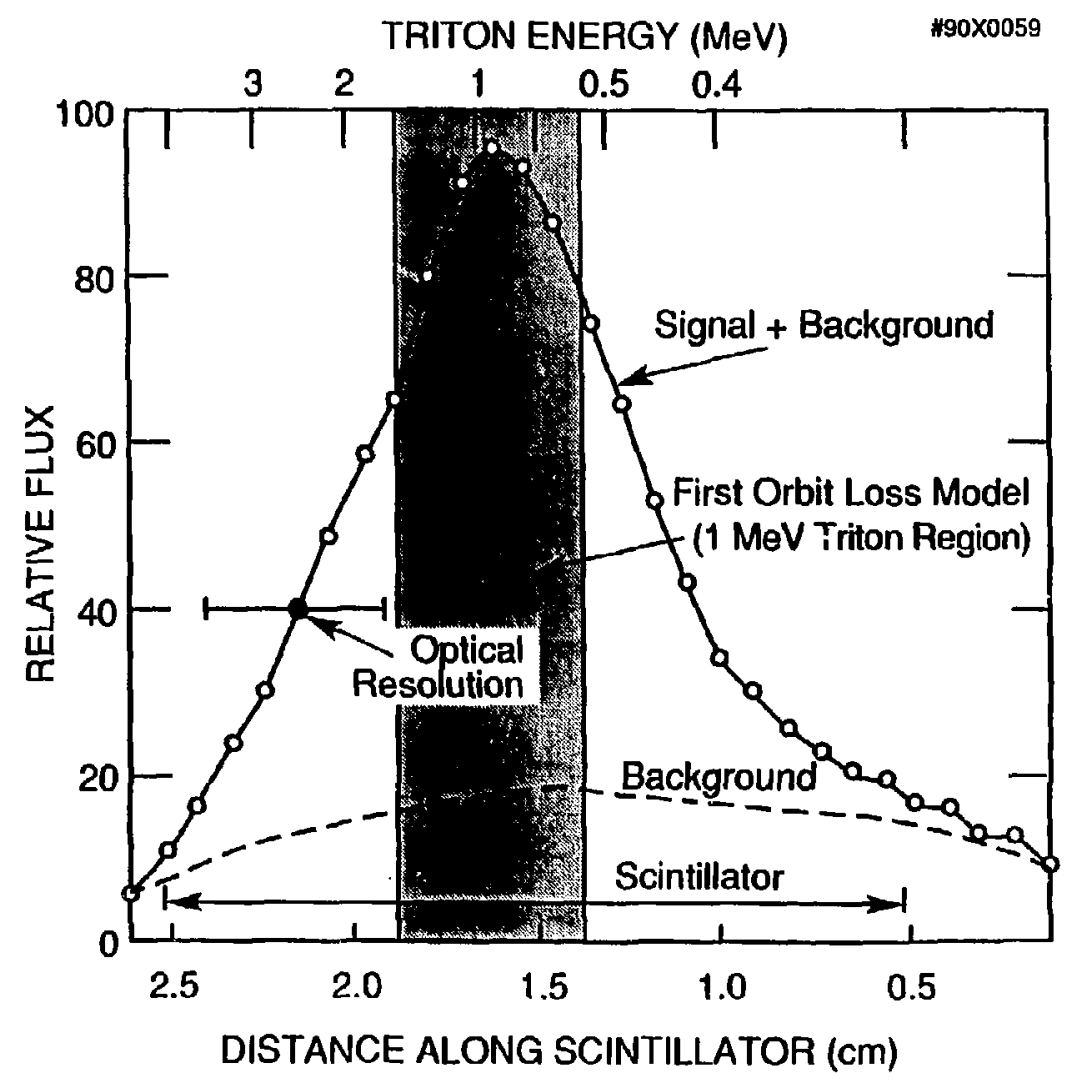


$109 \times 0593$

OREITS VS. PITCH ANGLE

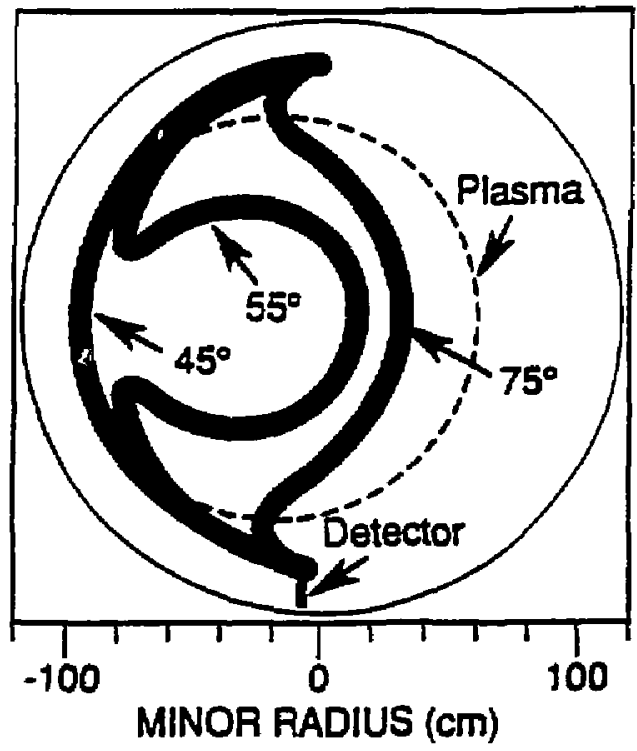

ORBITS VS. CURRENT

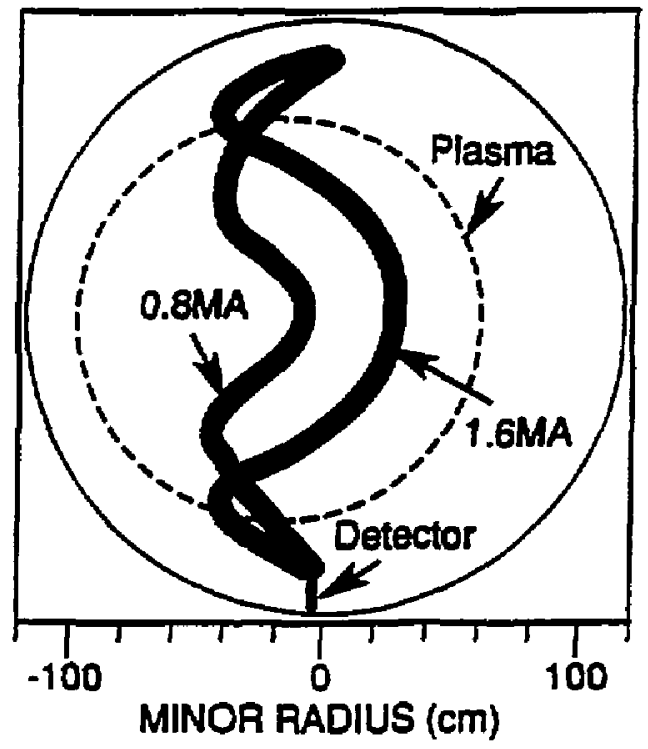

FIG. 7 


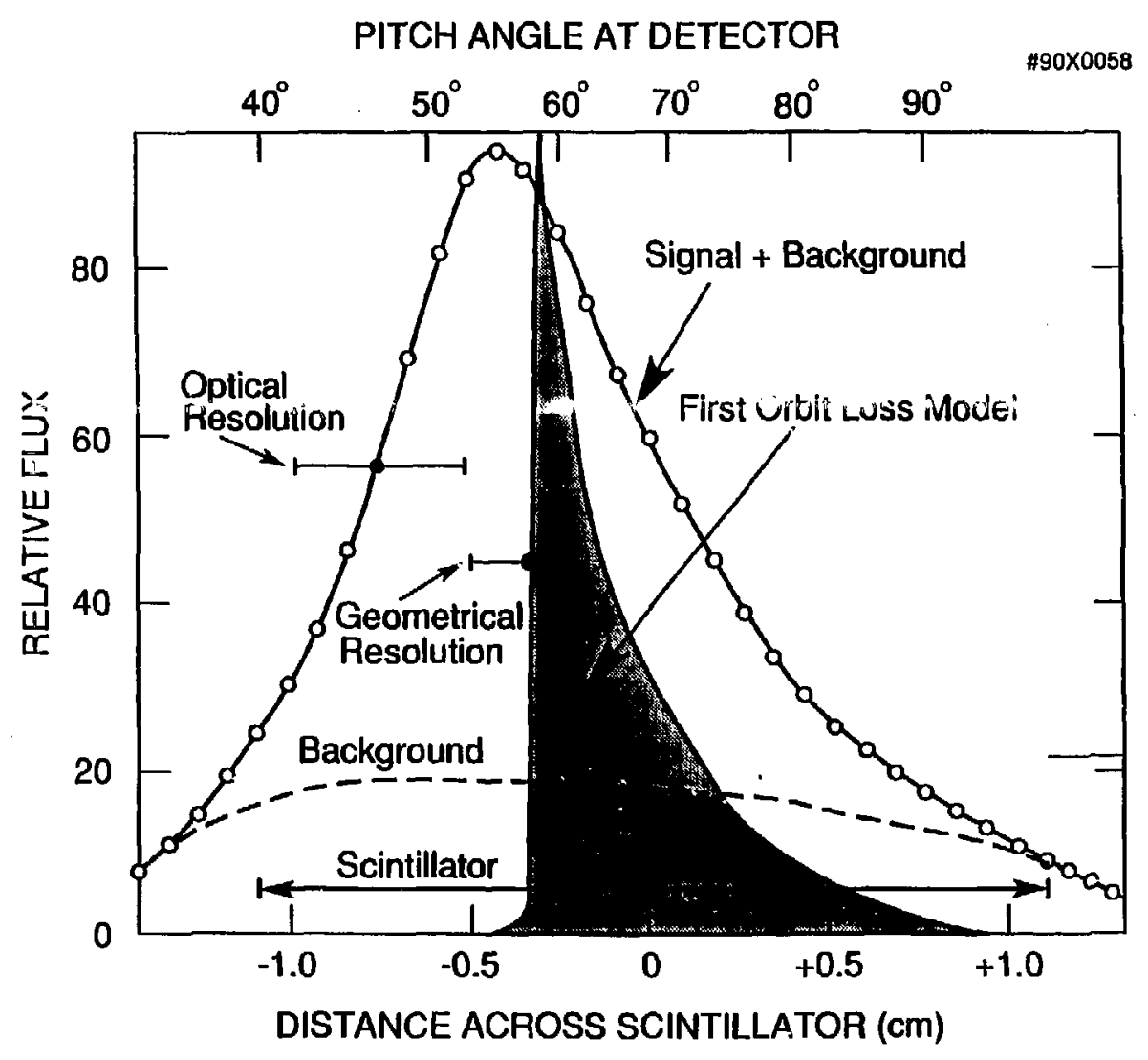


\#89X0488

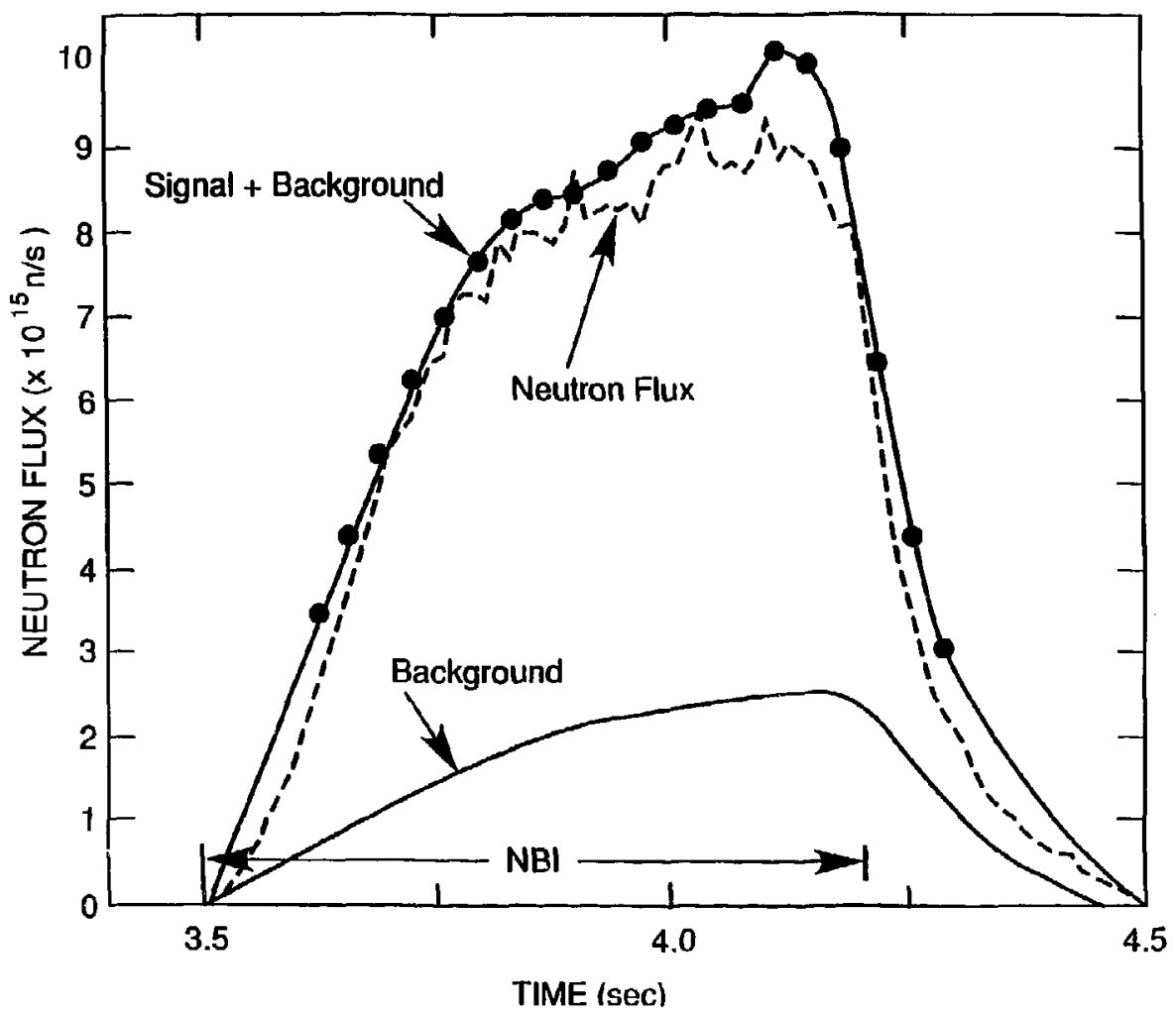




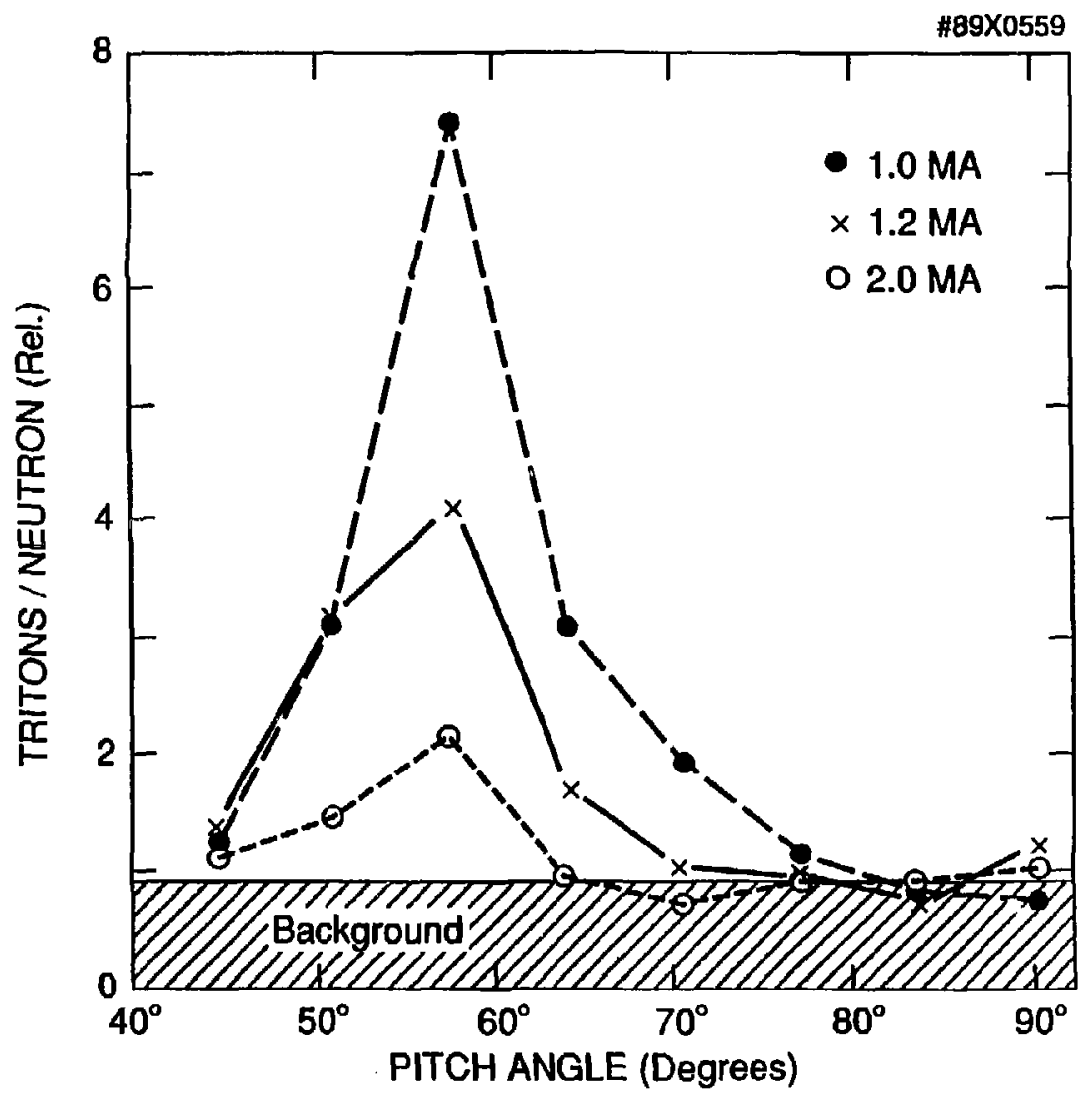

FIG. 10 
\#89X0591

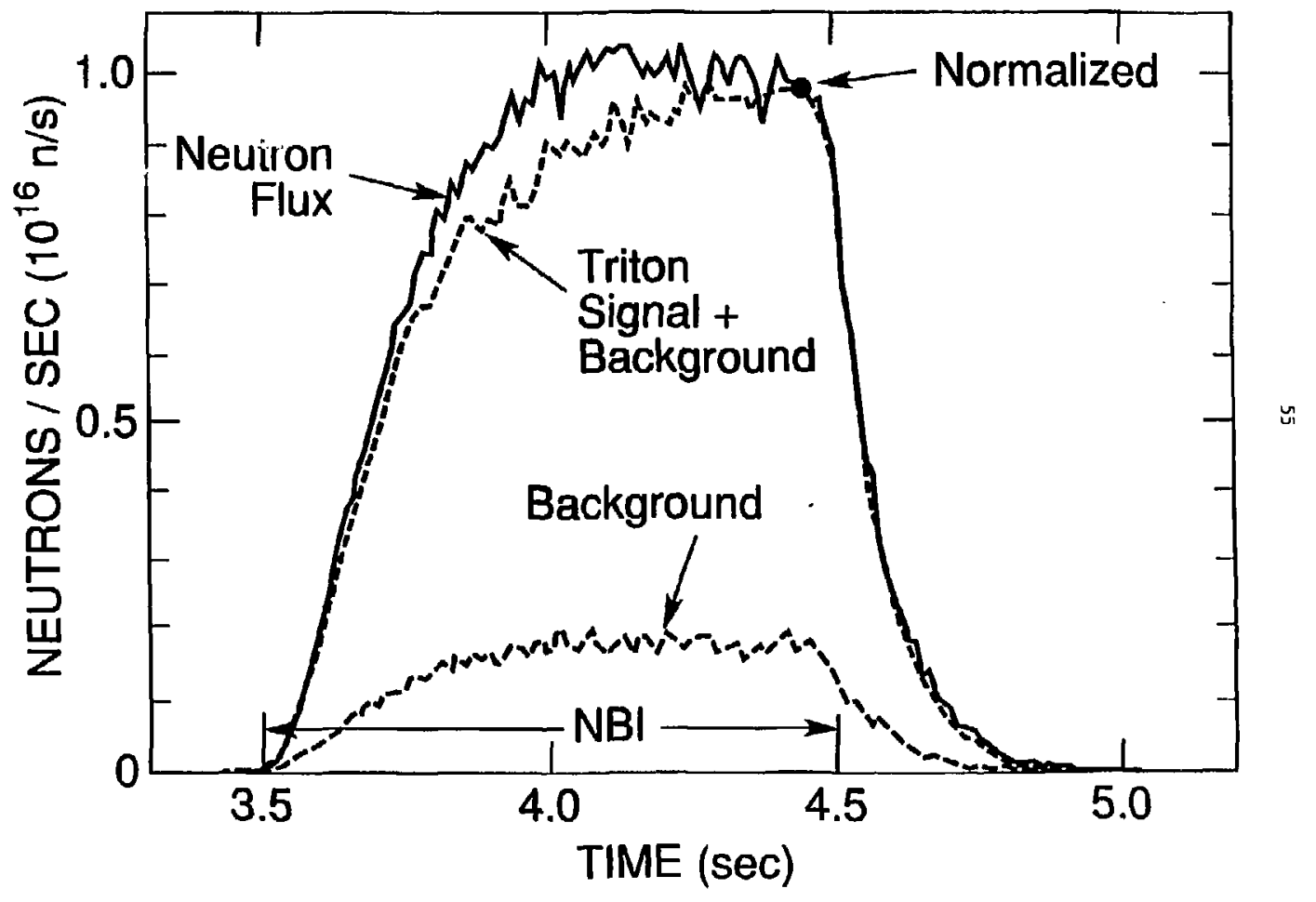

IfI (s. 11 

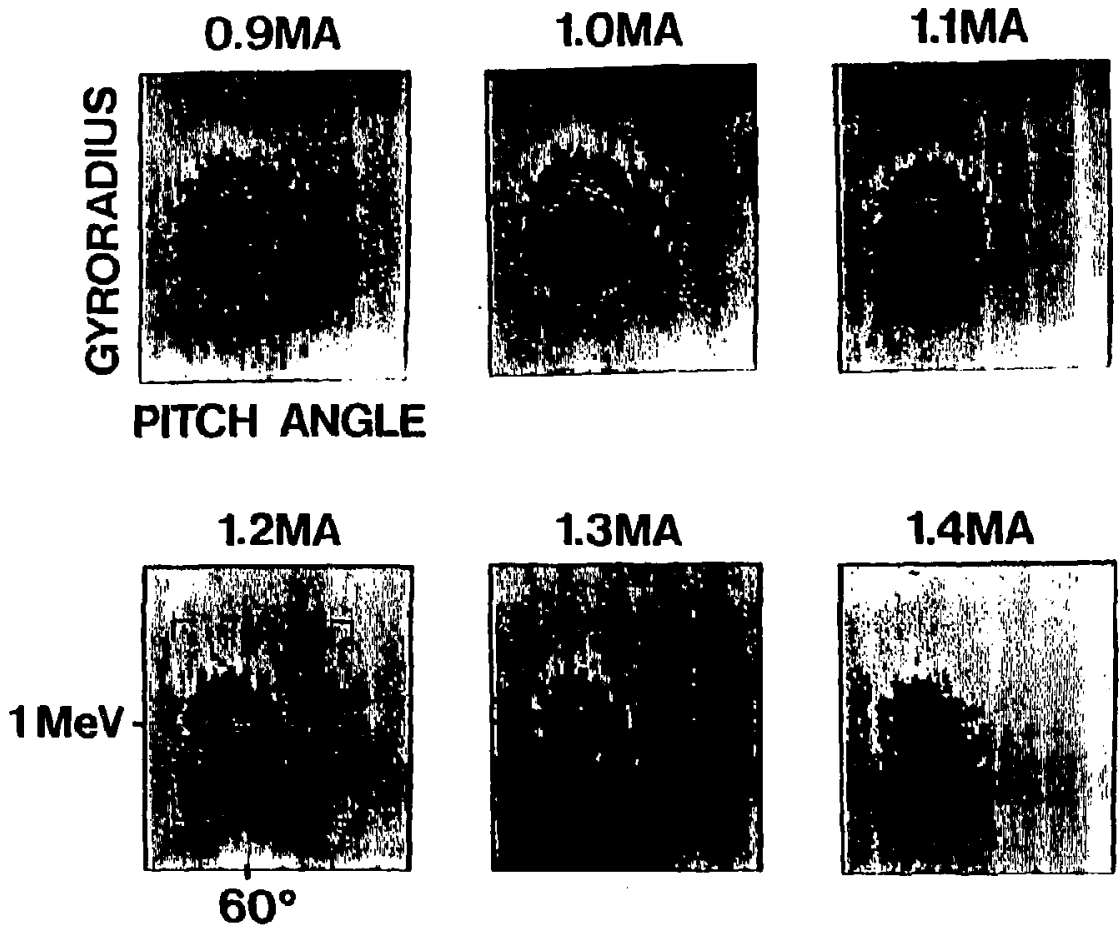


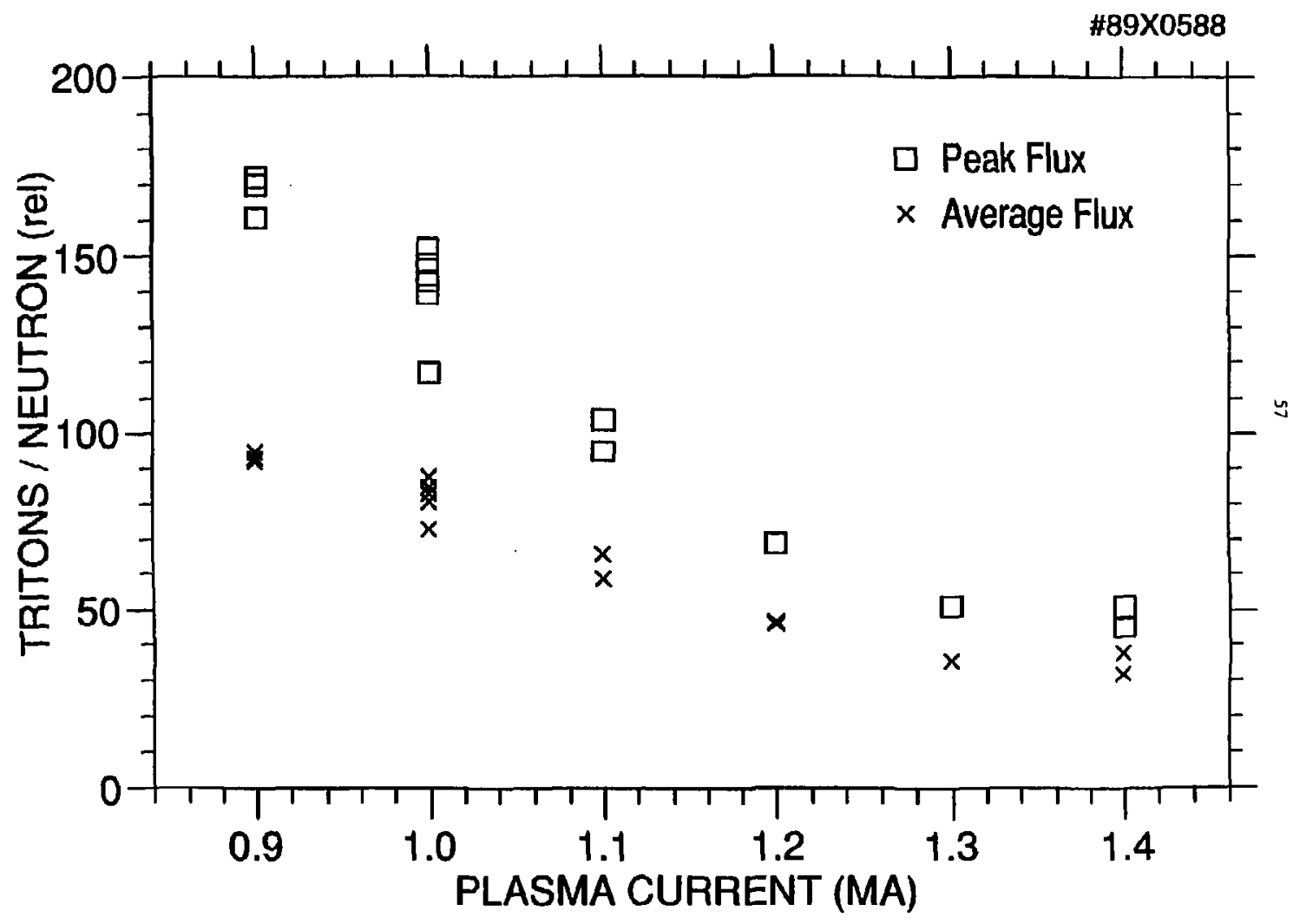

FTo, 13 


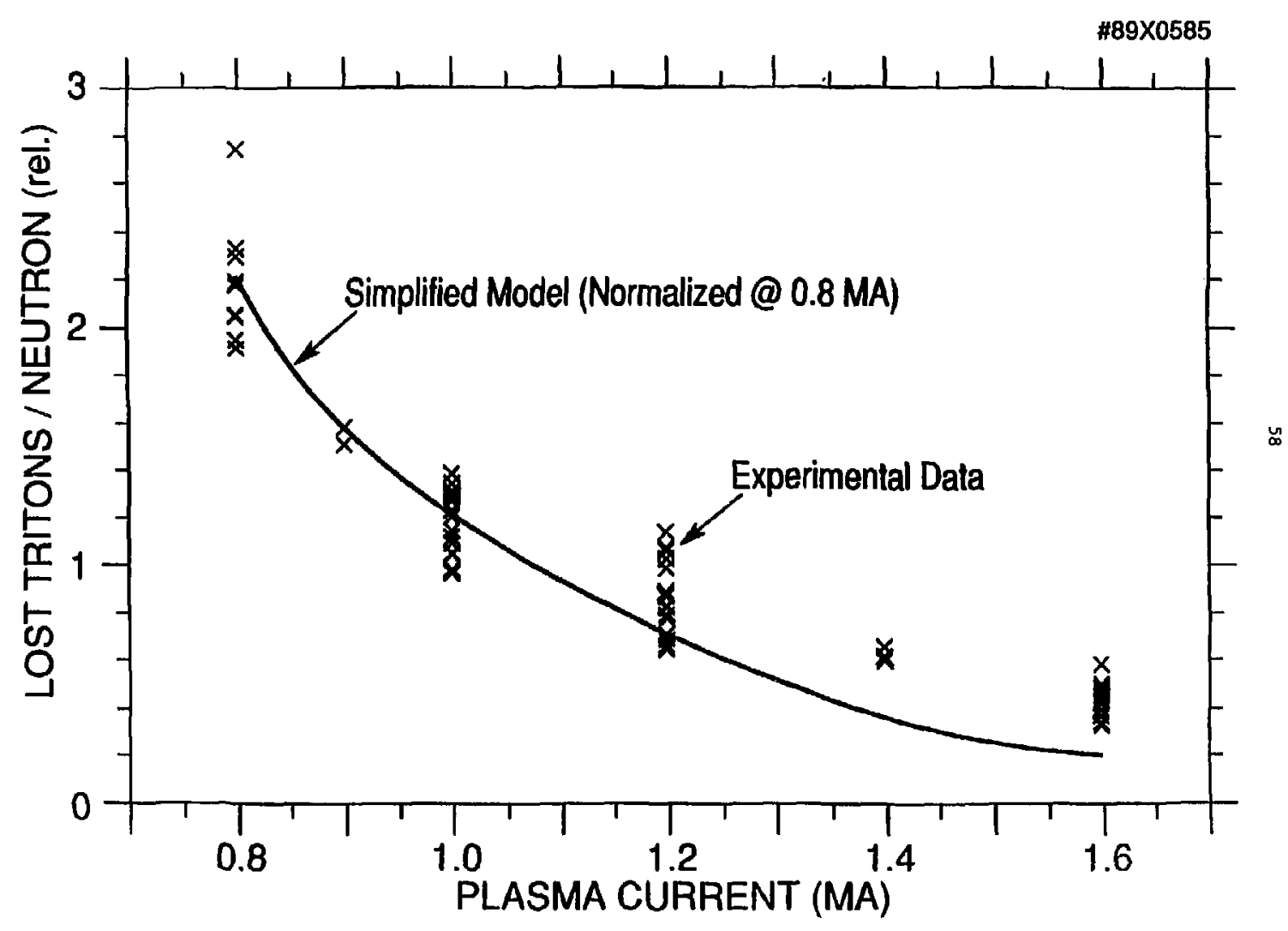

PII. $1 / 4$ 


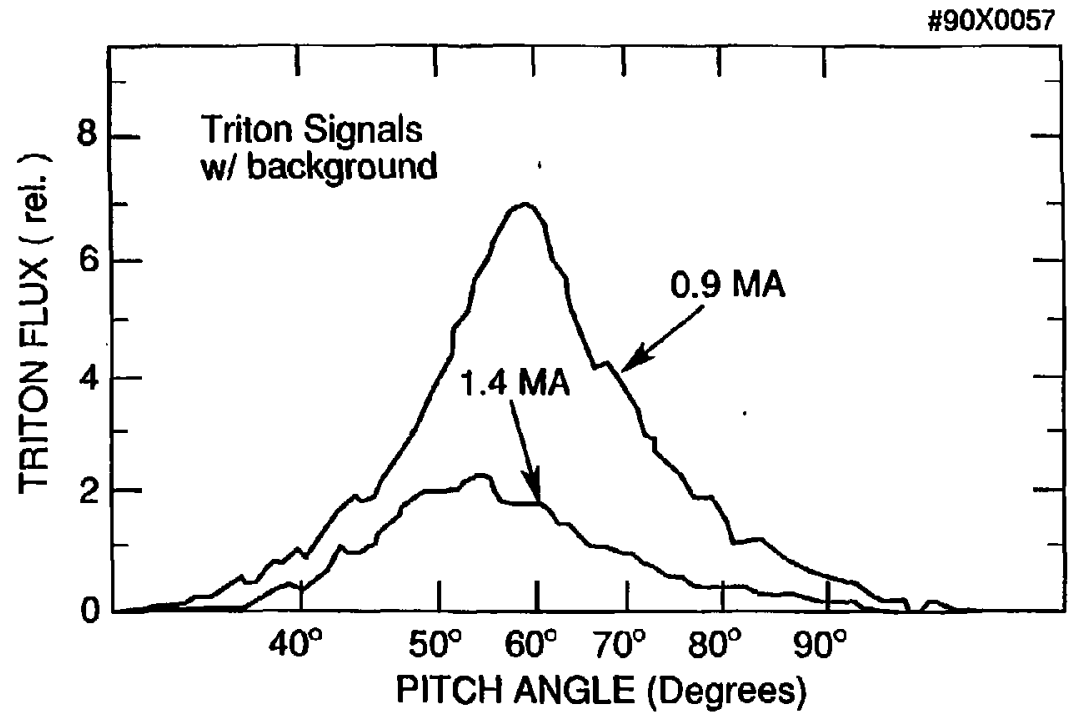




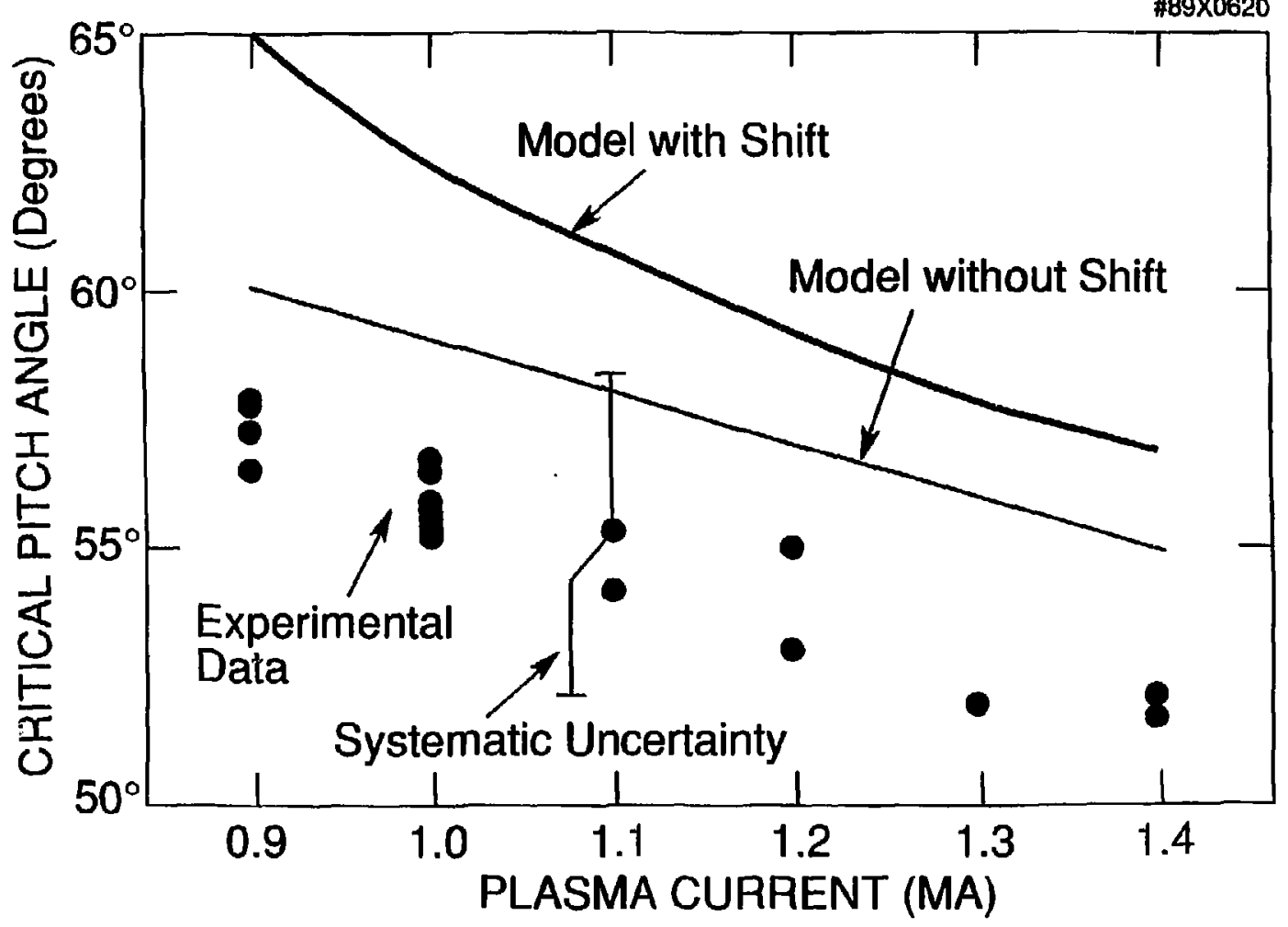




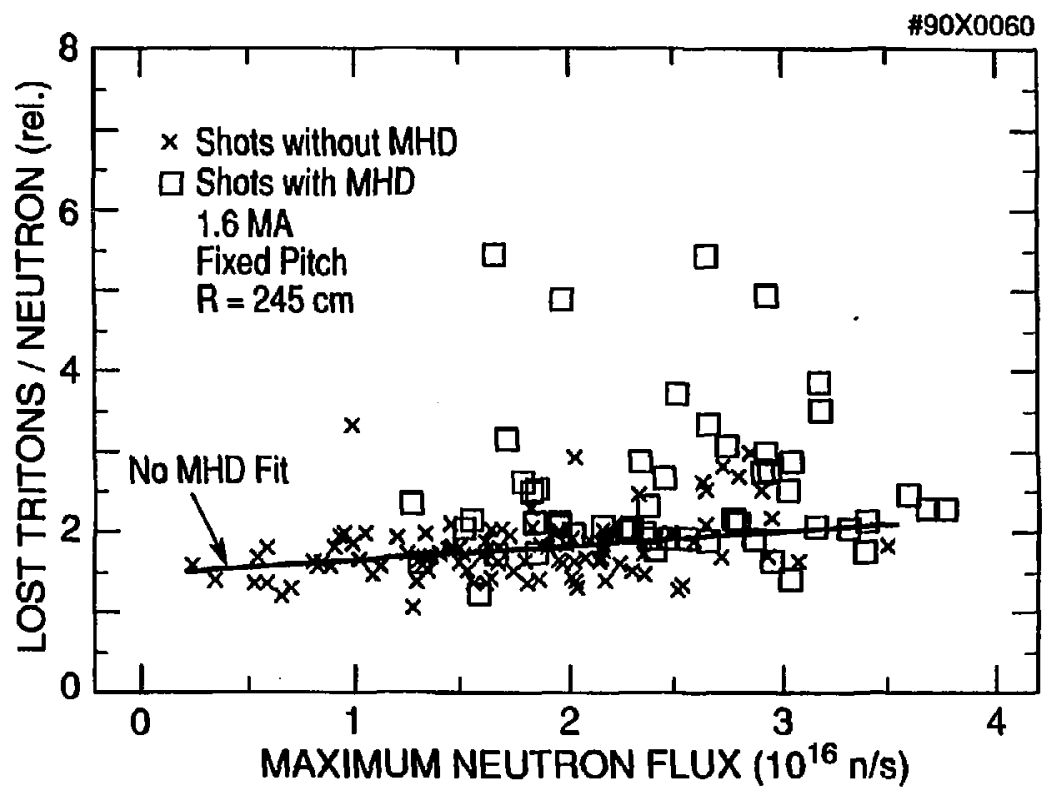




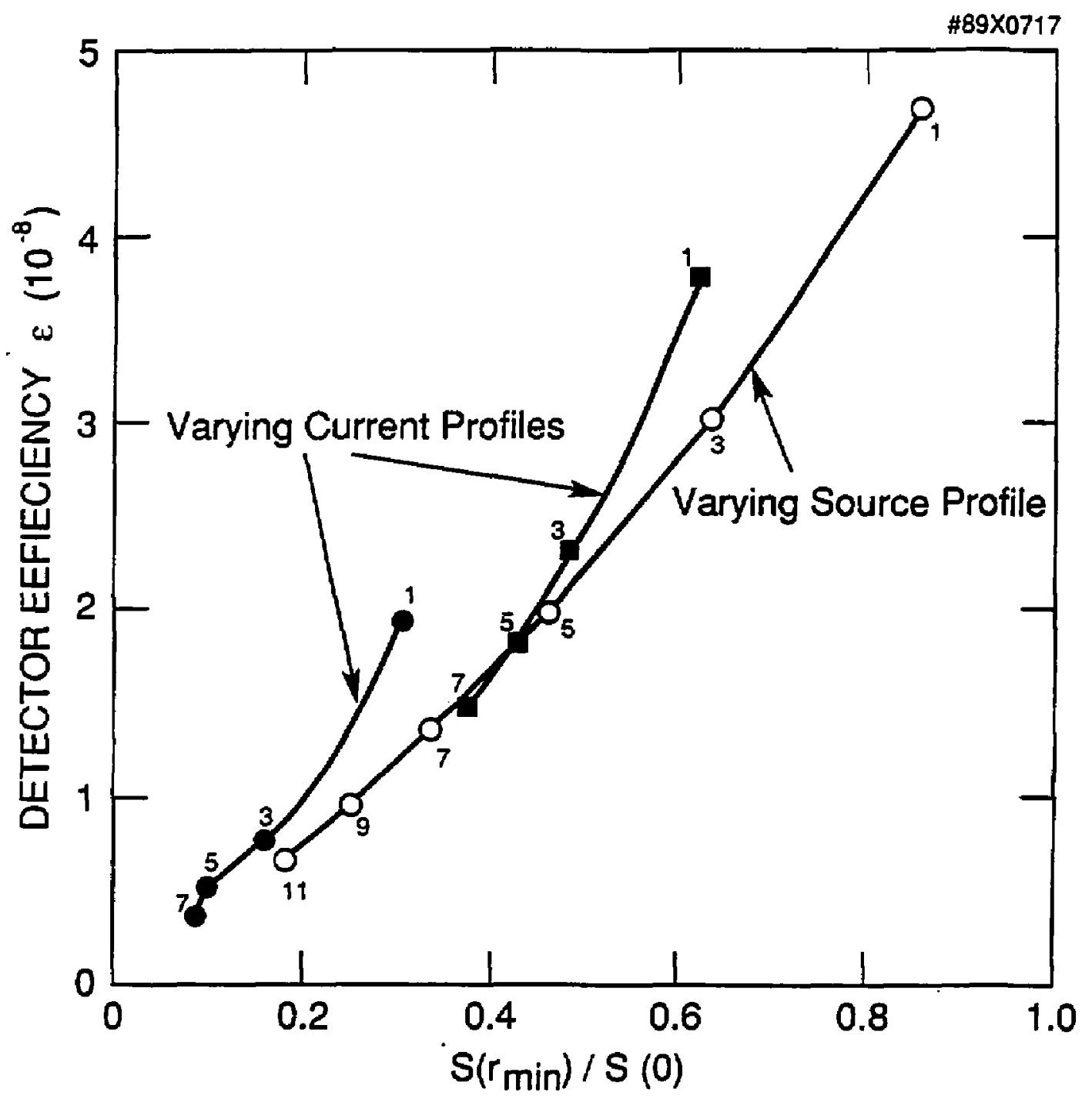

FIG. 18 
n90X0019

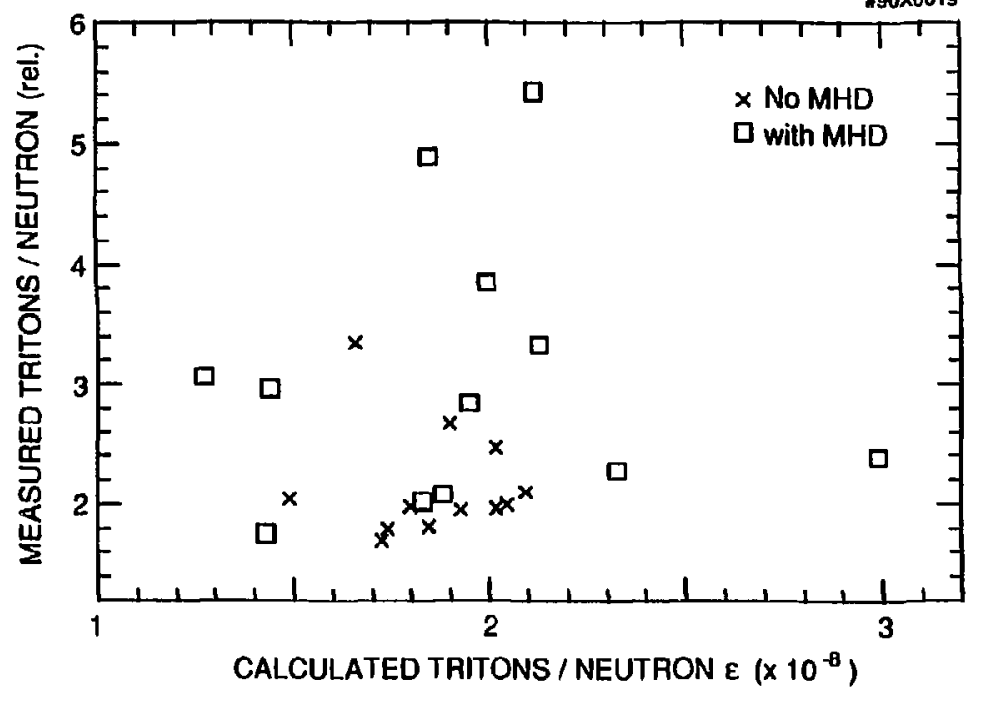

인

FJ6. 19 


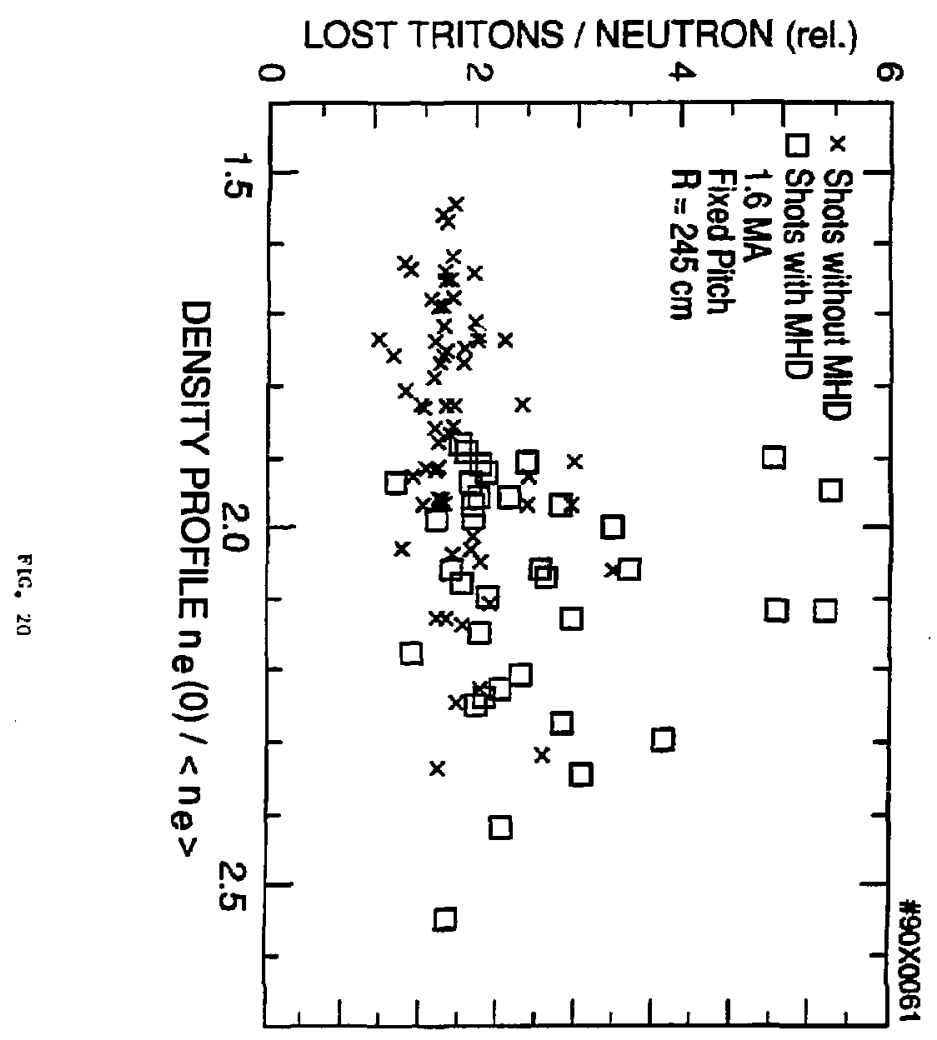




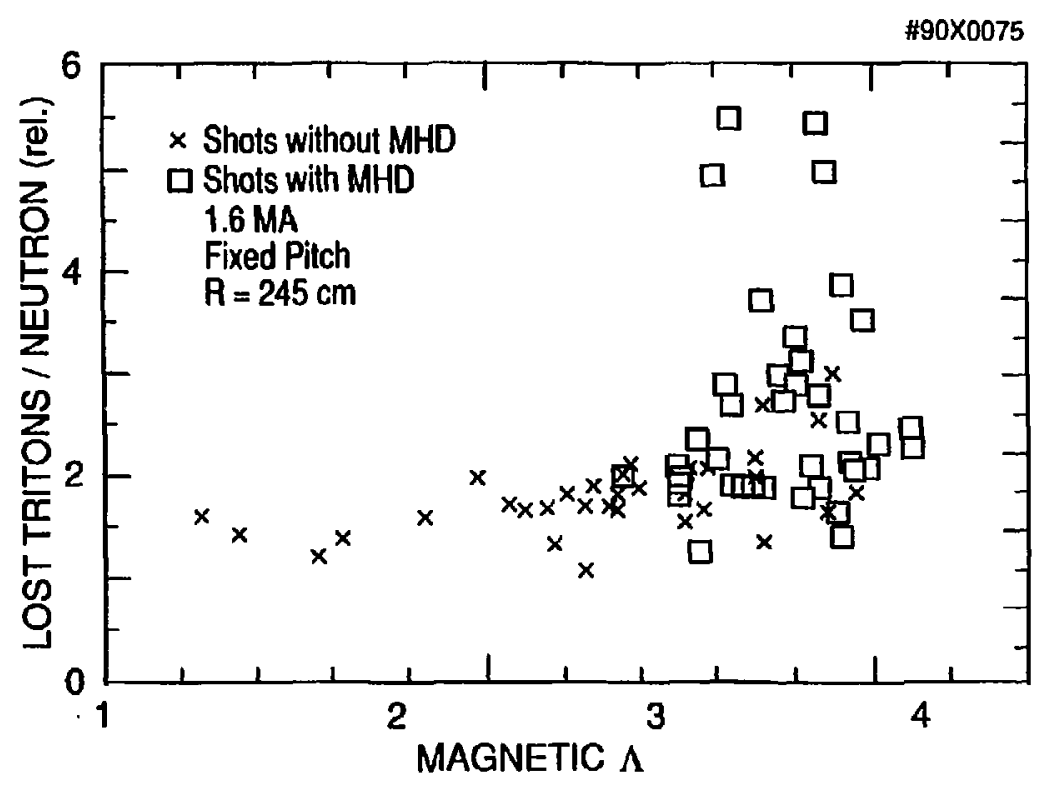




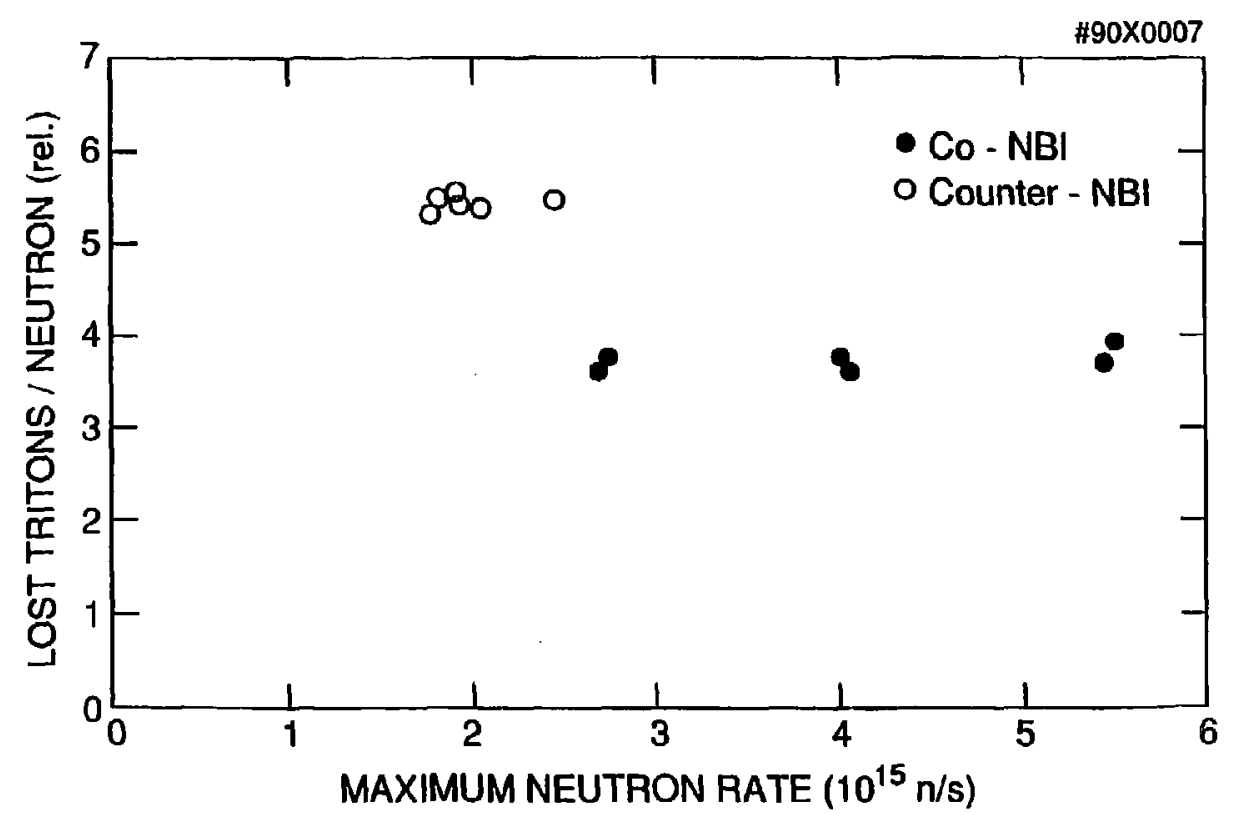




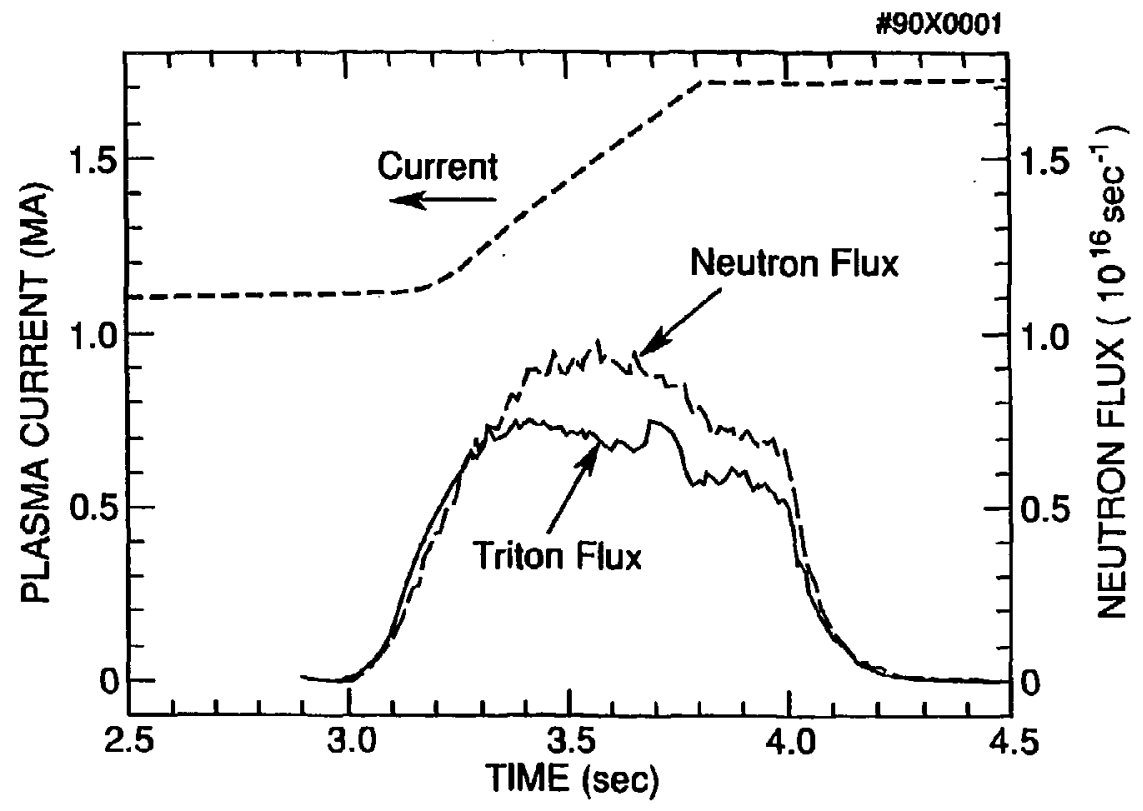

EIG. 23 


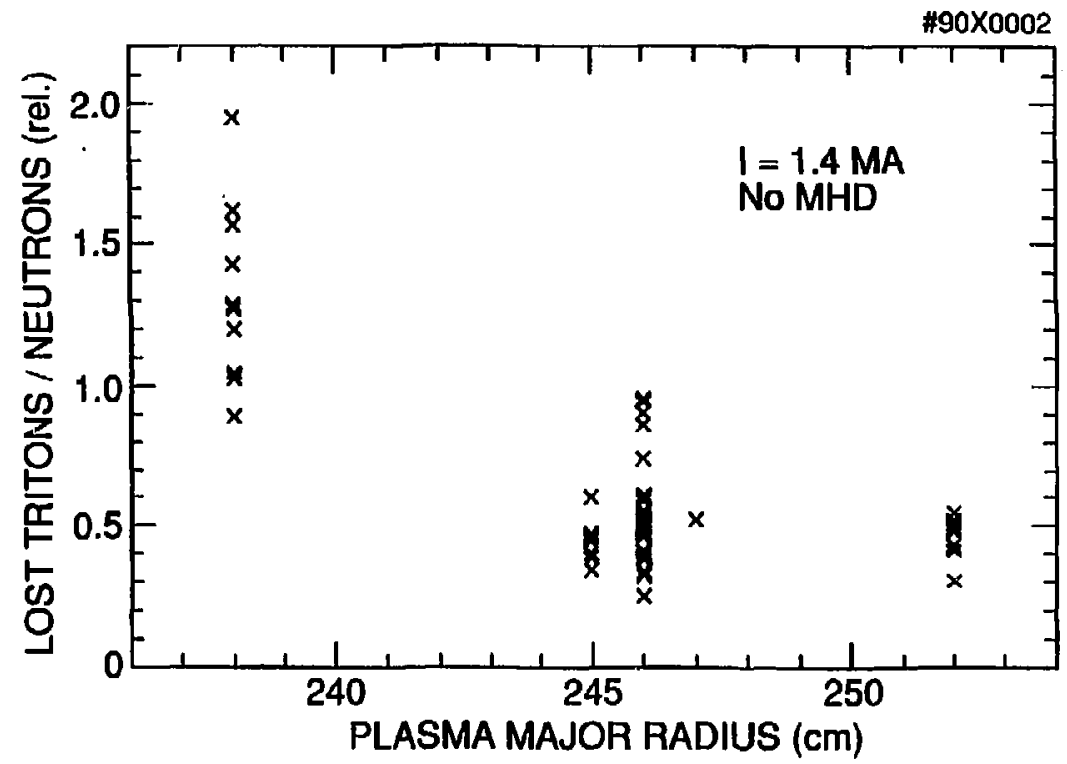




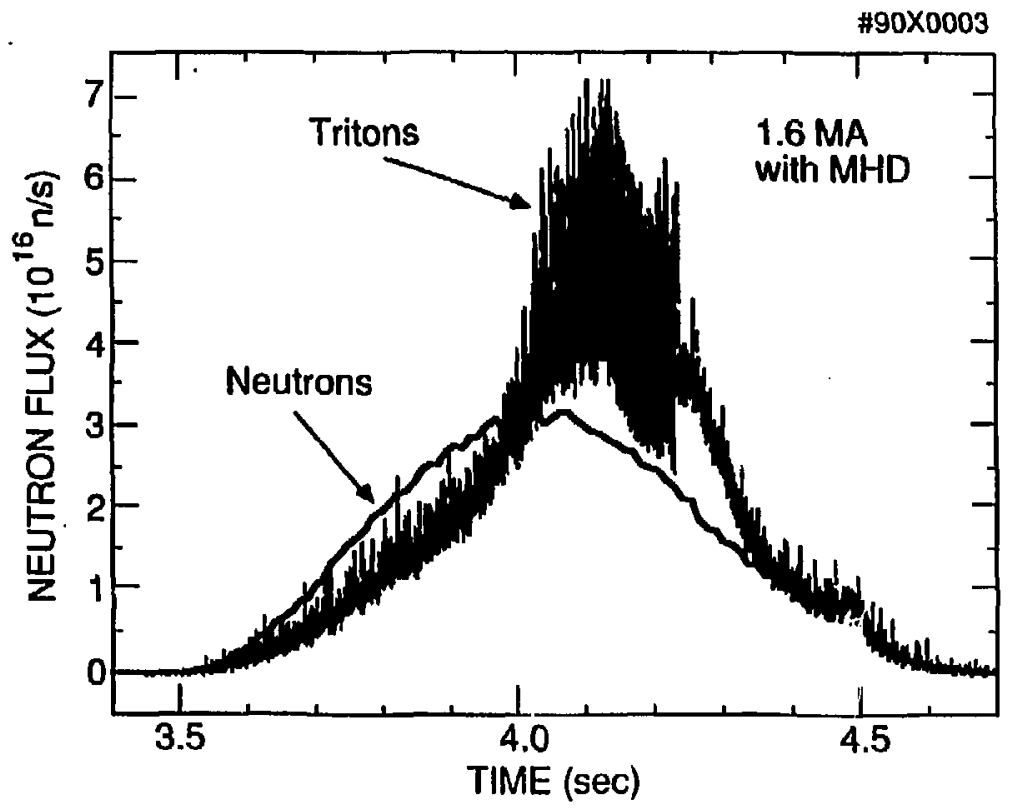

JIT. 25 


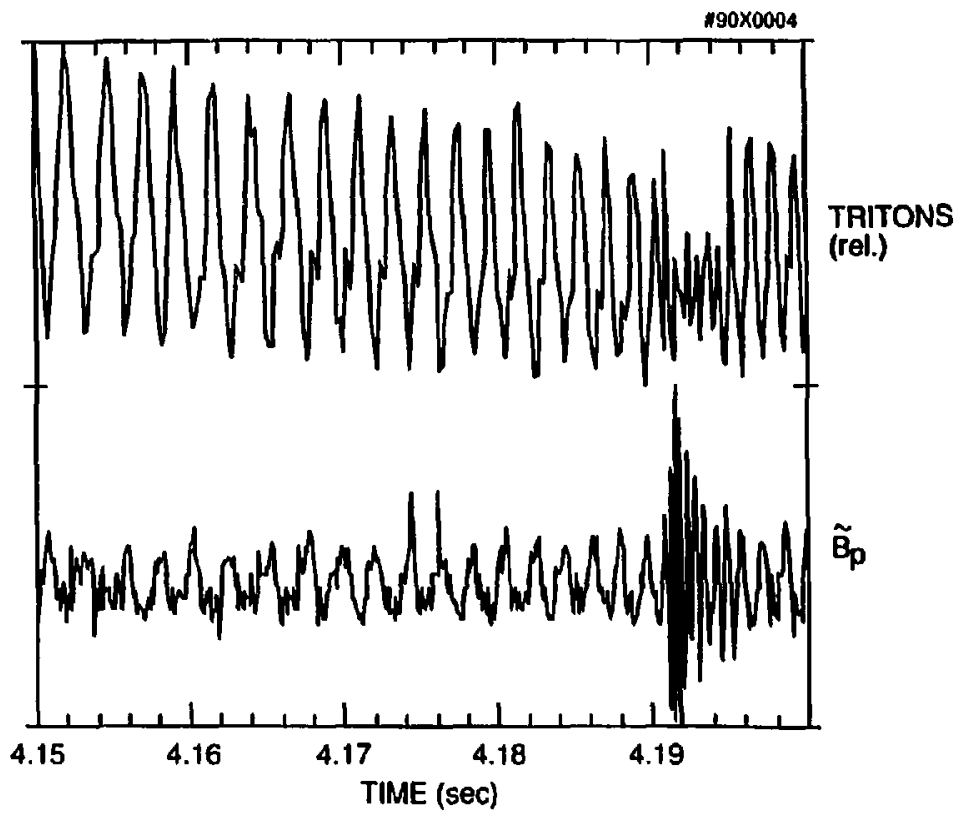

FIG. 26 


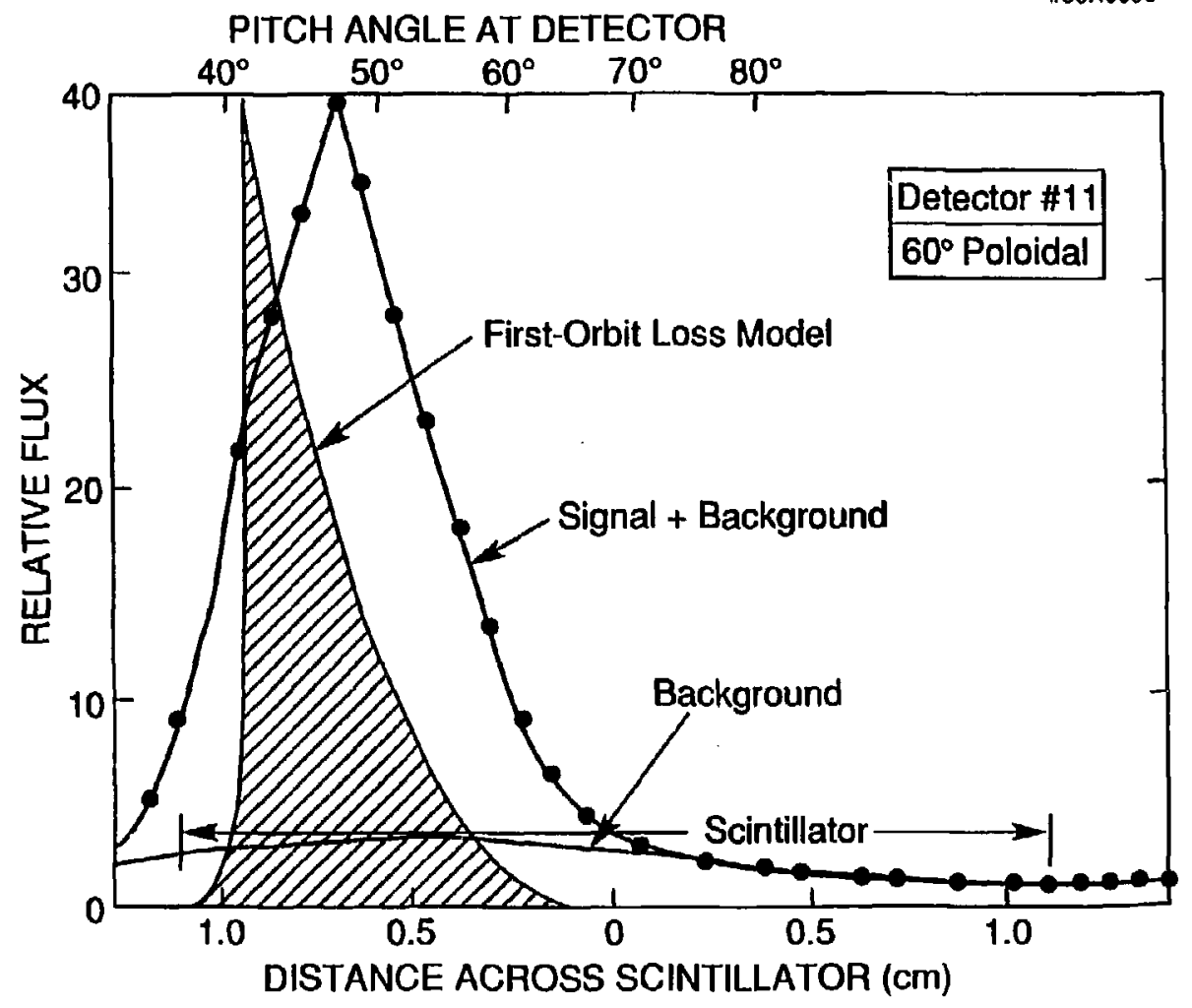




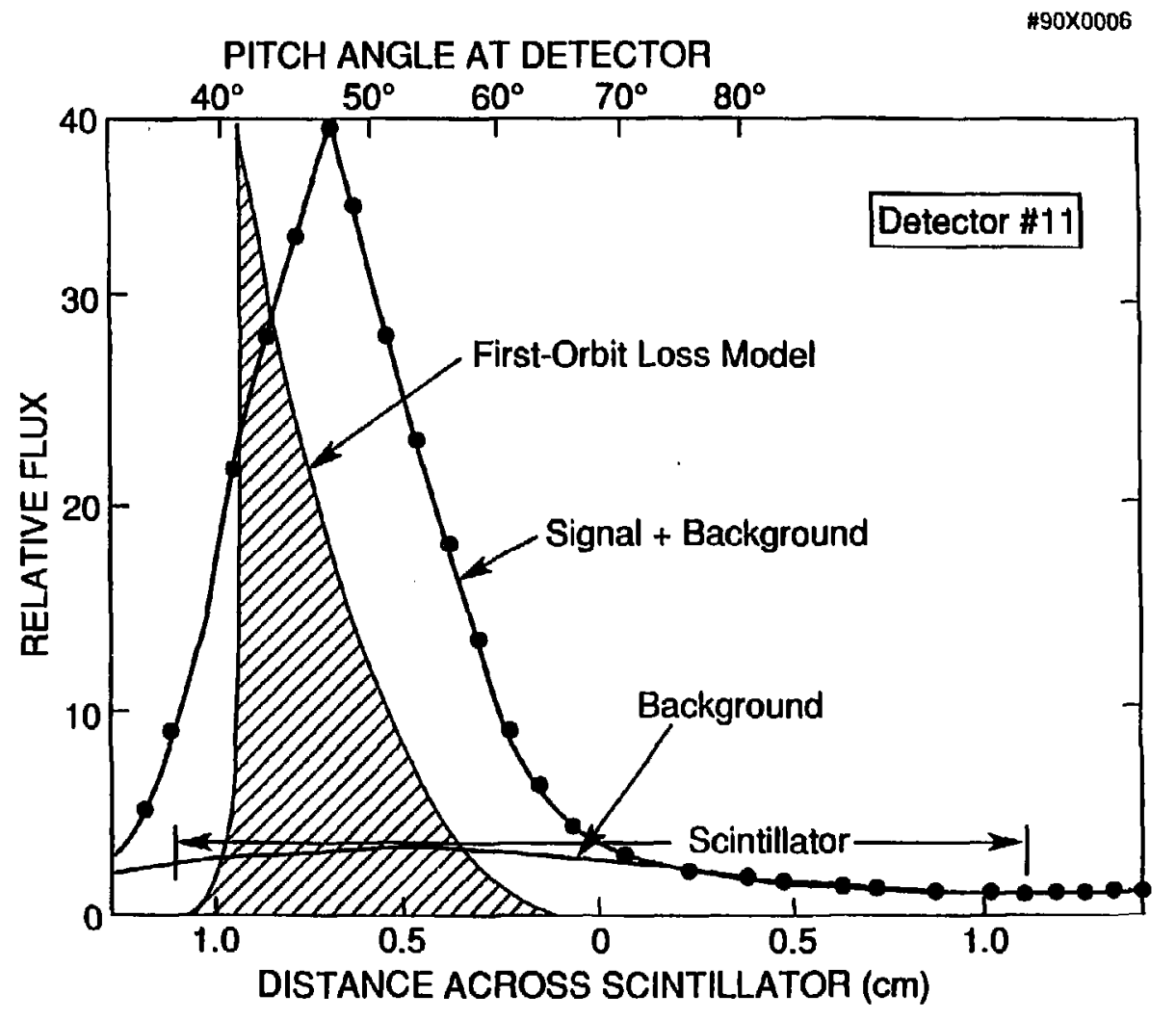

FIG, $2 k$ 